\title{
NASA Technology Area 07 Human Exploration Destination Systems Roadmap
}

\author{
Kriss J. Kennedy, architect ${ }^{1}$ \\ NASA TA07 HEDS Roadmap Team ${ }^{2}$
}

\begin{abstract}
This paper gives an overview of the National Aeronautics and Space Administration (NASA) Office of Chief Technologist (OCT) led Space Technology Roadmap definition efforts. This paper will given an executive summary of the technology area 07 (TA07) Human Exploration Destination Systems (HEDS). These are draft roadmaps being reviewed and updated by the National Research Council.

Deep-space human exploration missions will require many "game changing" technologies to enable safe missions, become more independent, and enable intelligent autonomous operations and take advantage of the local resources to become self-sufficient thereby meeting the goal of sustained human presence in space. Taking advantage of in-situ resources enhances and enables revolutionary robotic and human missions beyond the traditional mission architectures and launch vehicle capabilities. Mobility systems will include in-space flying, surface roving, and Extra-vehicular Activity/Extravehicular Robotics (EVA/EVR) mobility. These "push" missions will take advantage of sustainability and supportability technologies that will allow mission independence to conduct human mission operations either on or near the Earth, in deep space, in the vicinity of Mars, or on the Martian surface while opening up commercialization opportunities in low Earth orbit (LEO) for research, industrial development, academia, and entertainment space industries.

The Human Exploration Destination Systems (HEDS) Technology Area (TA) 7 Team has been chartered by the Office of the Chief Technologist (OCT) to strategically roadmap technology investments that will enable sustained human exploration and support NASA's missions and goals for at least the next 25 years. HEDS technologies will enable a sustained human presence for exploring destinations such as remote sites on Earth and beyond including, but not limited to, LaGrange points, low Earth orbit (LEO), high Earth orbit (HEO), geosynchronous orbit (GEO), the Moon, near-Earth objects (NEOs), which > 95\% are asteroidal bodies, Phobos, Deimos, Mars, and beyond. The HEDS technology roadmap will strategically guide NASA and other U.S. Government agency technology investments that will result in capabilities enabling human exploration missions to diverse destinations generating high returns on investments.
\end{abstract}

\footnotetext{
${ }^{1}$ NASA Johnson Space Center, 2101 Nasa Parkway, M/S EA351, Houston, TX 77058; 281.483.6629; kriss.j.kennedy@nasa.gov

${ }^{2}$ NASA TA07 HEDS Roadmap Team consists of Kriss Kennedy (TA07 Chair, JSC), Leslie Alexander - MSFC, Rob Landis - ARC, Diane Linne - GRC, Carole Mclemore - MSFC, Edgardo Santiago-Maldonado - KSC, David L. Brown - JSC
} 


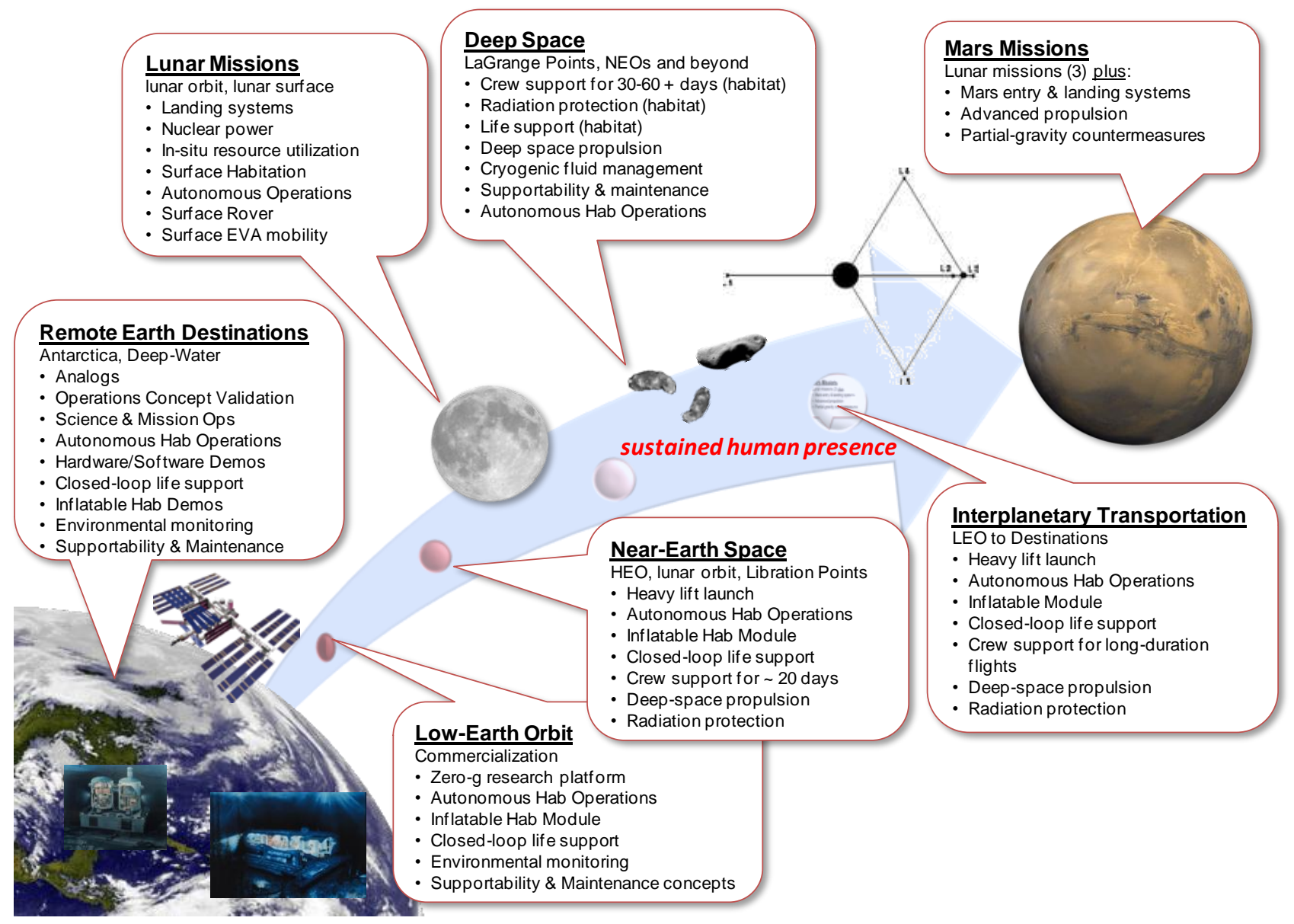

Figure 1: Human Exploration Destination Systems Enable Sustained Human Presence

The HEDS technology area focused on "fundamental" (Technology Readiness Level (TRL) 1-3) and "applied" (TRL 3-6) technologies that will enable a sustained human presence to a variety of destinations (figure 1). Human exploration destinations include deep-water ocean destinations in support of the evolutionary approach to deep-space destinations such as HEO, NEO, interplanetary travel, and planetary missions. HEDS technologies address both evolutionary and revolutionary game-changing technologies that will not only fulfill "pull" missions, but also enable new "push" missions. The HEDS technologies focus on enabling destination capabilities in an incremental "Block" five-year (5 yr) cycle of maturation. Each "block" builds on the previous technologies and adds additional capabilities.

The TA7 HEDS Technology Area Breakdown Structure (TABS) is divided into six Level-2 technology focus areas. Figure 2 illustrates the TABS divisions as: 7.1 In-Situ Resource Utilization; 7.2 Sustainability and Supportability; 7.3 Advanced Human Mobility Systems; 7.4 Advanced Habitat Systems; 7.5 Mission Operations and Safety; and 7.6 Cross-Cutting Systems. 


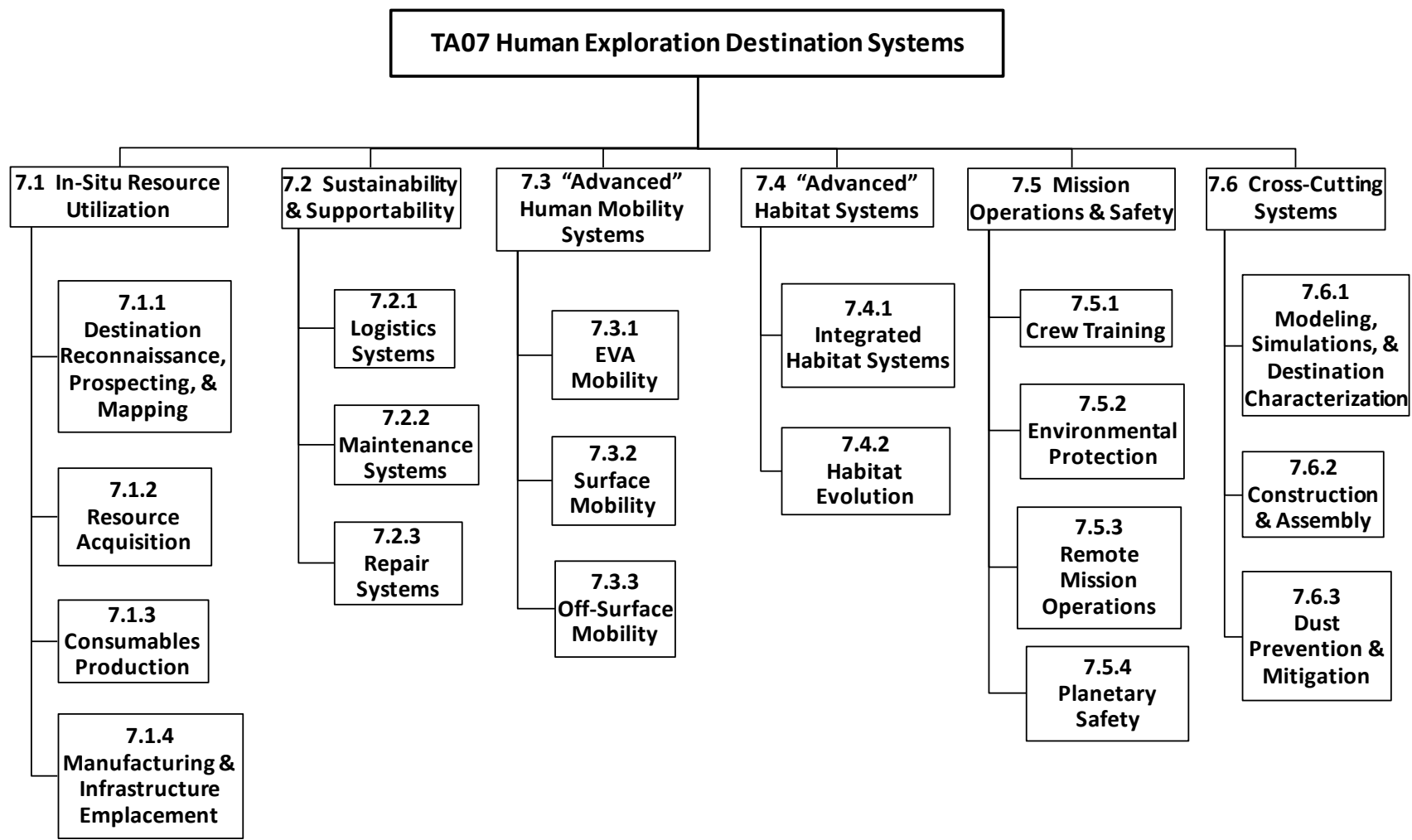

Figure 2: TA7 HEDS Technology Area Breakdown Structure

The HEDS TABS maps the respective fundamental and applied technologies to the four NASA Mission Directorates' pull missions as applicable. The applicable "pull-mission" is shown at the top of the roadmaps indicated as a "triangle" $(\mathbf{\Delta})$ symbol. Each of the Mission Directorates (Space Operations Mission Directorate (SOMD), Exploration Systems Mission Directorate (ESMD), Science Mission Directorate (SMD), and Aeronautics Research Mission Directorate (ARMD)) is identified by their respective color. "Push" mission opportunities are identified by the HEDS Team's developed Mission Capabilities Milestones "swim lane", and technology areas are indicated by a star (岤) symbol on the HEDS roadmaps shown in figure 3. The HEDS milestones shown on the roadmaps indicate the timeframe that the hardware and/or software should mature to a Technology Readiness Level 6 (TRL-6) level. This is based upon a mission PDR assuming launch minus four years (L-4) for robotic missions and launch minus six years (L-6) for human missions. Thus, if a human mission identified by a Mission Directorate is 2020, then the TRL6 would need to be matured by 2014. 


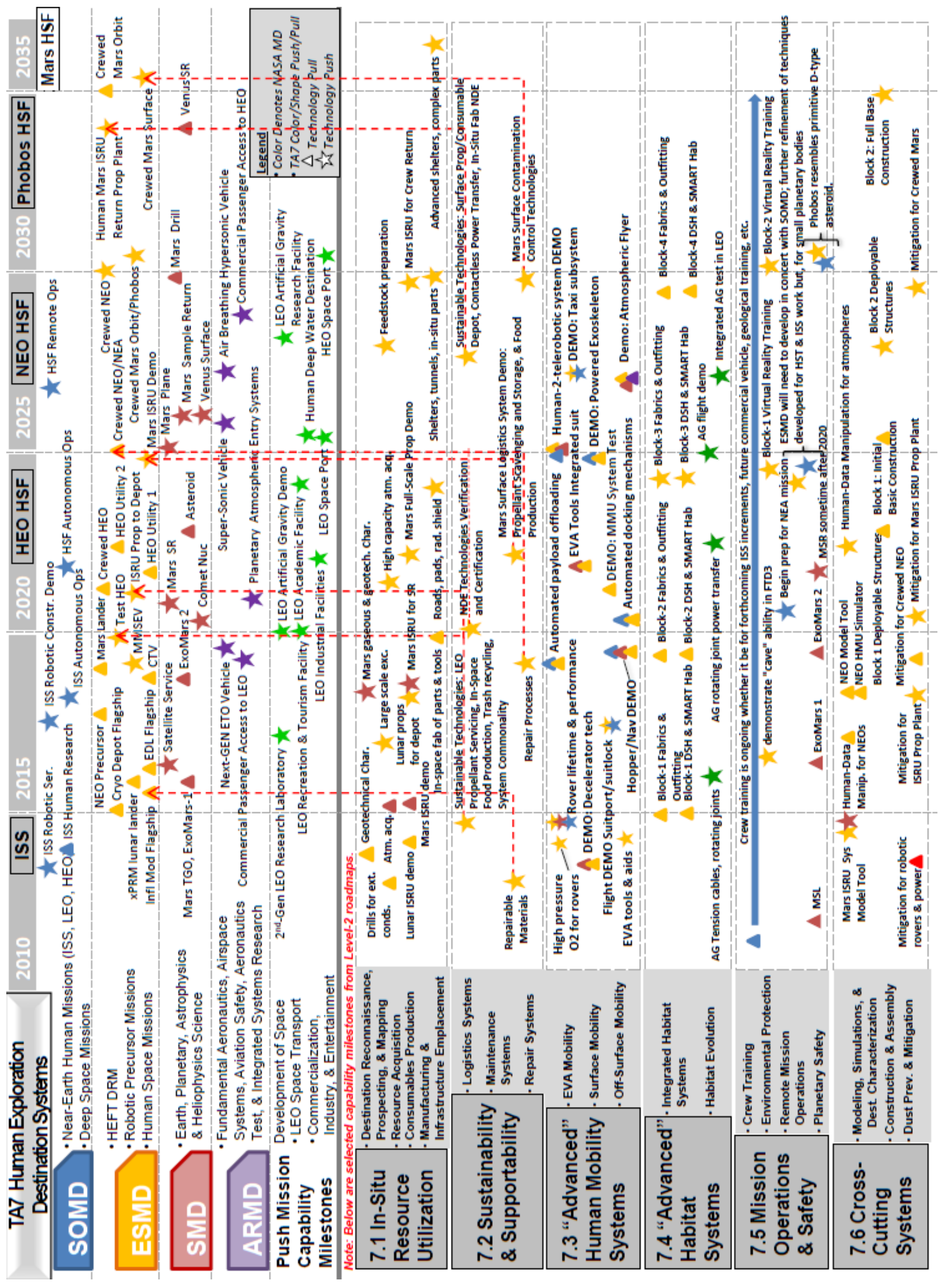

Figure 3: Human Exploration Destination Systems Level-1 Technology Area Strategic Roadmap 
Key words: Space Technology Roadmap, roadmap, Human Exploration Destination System, technology 


\title{
NASA Technology Area 07 Human Exploration Destination Systems Roadmap
}

\author{
Kriss J. Kennedy ${ }^{1}$ \\ NASA Johnson Space Center, Houston, TX 77058 \\ NASA OCT TA07 Roadmap Team ${ }^{2,3}$
}

\begin{abstract}
I. Abstract
This paper gives an overview of the National Aeronautics and Space Administration (NASA) Office of the Chief Technologist (OCT) led Space Technology Roadmap definition efforts. These draft roadmaps are currently being reviewed and updated by the National Research Council. This paper will provide an executive summary of the technology area 07 (TA07) Human Exploration Destination Systems (HEDS). Deep-space human exploration missions will require many "game changing" technologies to enable safe missions and to enable intelligent autonomous operations. These technologies will take advantage of the local resources to enable self-sufficiency, thereby, meeting the goal of sustained human presence in space. Taking advantage of in-situ resources enhances and enables revolutionary robotic and human missions beyond the traditional mission architectures and launch vehicle capabilities. Mobility systems used in these missions will include in-space flying, surface roving, and Extravehicular Activity/Extravehicular Robotics (EVA/EVR) mobility. The HEDS roadmap identifies "push" missions that will take advantage of sustainable and supportable technologies to conduct human mission operations either on or near the Earth, in deep space, in the vicinity of Mars, or on the Martian surface while opening up commercialization opportunities in low Earth orbit (LEO) for research, industrial development, academia, and entertainment space industries.

The HEDS TA07 Team has been chartered by the OCT to strategically roadmap technology investments that will enable sustained human exploration and support NASA's missions and goals for at least the next 25 years. HEDS technologies will enable a sustained human presence for exploring remote sites on Earth and destinations beyond Earth including, but not limited to, LaGrange points, low Earth orbit (LEO), high Earth orbit (HEO), geosynchronous orbit (GEO), the Moon, near-Earth objects (NEOs), Phobos, Deimos, Mars, and beyond. The HEDS technology roadmap guides NASA and other U.S. Government agency technology investments to areas of highest return while resulting in capabilities that support human exploration missions to diverse destinations. [I would move this up to the first part of the abstract to follow your first paragraph. Some of the information in your current $2^{\text {nd }}$ paragraph is duplicative. Might want to cut some of that out or highlight other things like some of the technologies under HEDS.]
\end{abstract}

\section{Introduction}

Deep-space human exploration missions will require many "game changing" technologies to enable safe missions with intelligent autonomous operations, utilizing local resources to become self-sufficient, thereby meeting the goal of sustained human presence in space. Taking advantage of in-situ resources enhances and enables revolutionary robotic and human missions beyond the traditional mission architectures and launch vehicle capabilities by reducing overall mass of supplies launched from Earth and thus the number of launches per mission - a net cost savings. Mobility systems will include in-space flying, surface roving, and EVA/EVR mobility. These "push" missions will take advantage of sustainability and supportability technologies that will allow mission independence to conduct human mission operations either on or near the Earth, in deep space, in the vicinity of Mars, or on the Martian surface while opening up commercialization opportunities in LEO for research, industrial development, academia, and entertainment space industries.

\footnotetext{
${ }^{1}$ Architect, NASA Johnson Space Center, 2101 NASA Parkway, M/S EA351, Houston, TX 77058; +1.281.483.6629; kriss.j.kennedy@nasa.gov

${ }^{2}$ NASA TA07 HEDS Core Team consists of Kriss Kennedy (Chair,/JSC); Leslie Alexander/MSFC; Rob Landis/ formerly ARC, now GSFC/WFF; Diane Linne/GRC; Carole Mclemore/MSFC; Edgardo Santiago-Maldonado/KSC

${ }^{3}$ NASA TA07 HEDS Support Team consists of David Brown/JSC; Peter Curreri/MSFC; Peggy Guirgis/JSC; Michael Lowry/ARC; John Sims/JSC;
} 


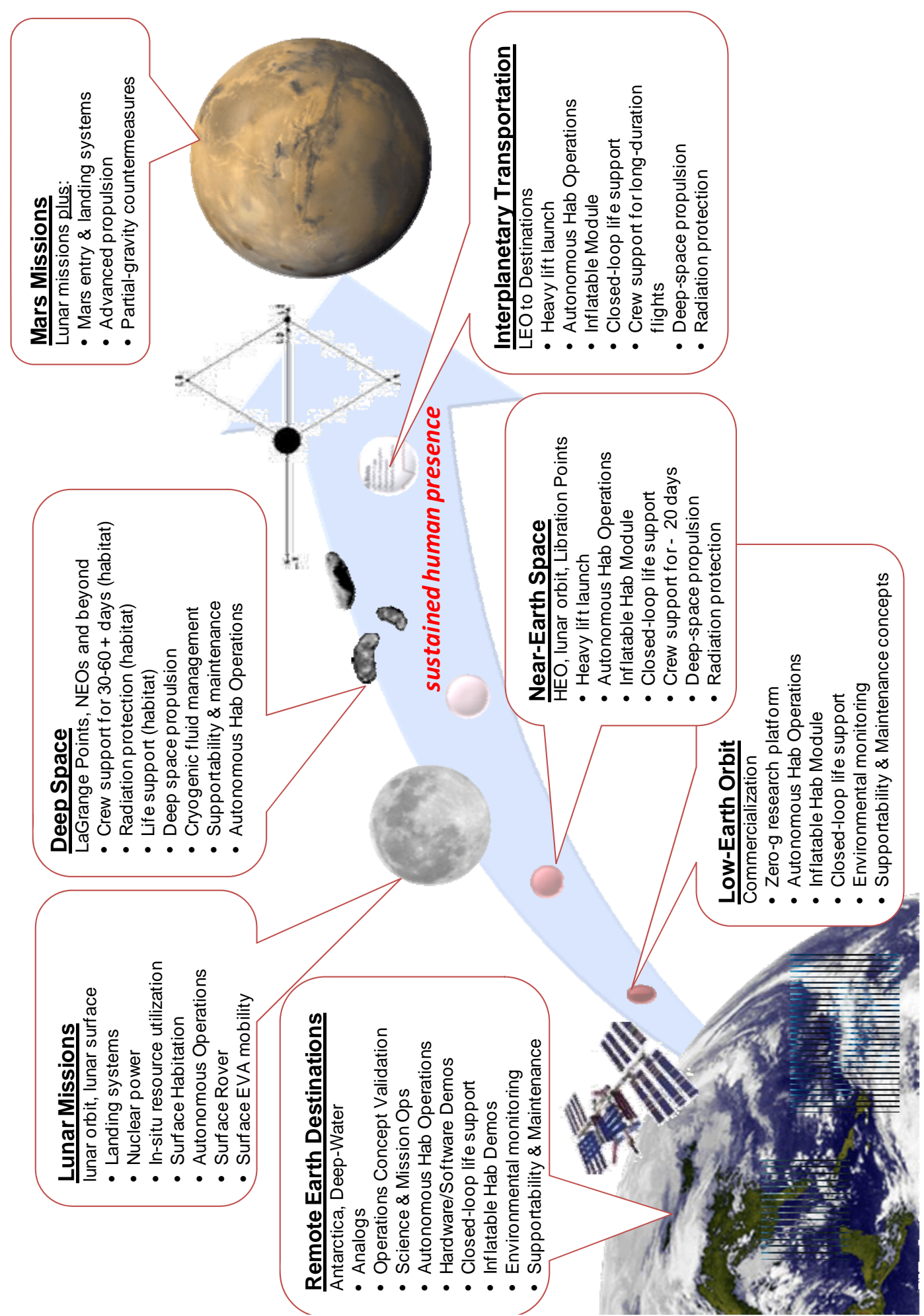

Figure 1: Human Exploration Destination Systems Enable Sustained Human Presence

The HEDS Technology Area TA07 Team has been chartered by the OCT to develop a strategic roadmap of technology investments that will enable sustained human exploration and support NASA's missions and goals for at least the next 25 years. HEDS technologies will enable a sustained human presence for exploring destinations such as remote sites on Earth and beyond including, but not limited to, LaGrange points, low Earth orbit (LEO), high Earth orbit (HEO), geosynchronous orbit (GEO), the Moon, near-Earth objects (NEOs), which $>95 \%$ are asteroidal 
bodies, Phobos, Deimos, Mars, and beyond. The HEDS technology roadmap will strategically guide NASA and other U.S. Government agency technology investments that will result in capabilities enabling human exploration missions to diverse destinations generating high returns on investments.

The HEDS technology area focused on "fundamental" (Technology Readiness Level (TRL) 1-3) and "applied" (TRL 3-6) technologies that will enable a sustained human presence to a variety of destinations (figure 1). Human exploration destinations include those destinations supporting an evolutionary approach of deep-space exploration such as HEO, NEO, interplanetary travel, and planetary missions. HEDS technologies address both evolutionary and revolutionary game-changing technologies that will not only fulfill "pull" missions, but also enable new "push" missions such as ISRU supplied Fuel Depots at L1 or self-sustained human presence on Mars surface. The HEDS roadmaps capture technologies that provide destination capabilities in an incremental "Block", or five year cycle, of maturation. Each "block" builds on the previous one, adding additional capabilities.

The TA07 HEDS Technology Area Breakdown Structure (TABS) consist of six Level-2 technology focus areas. Figure 2 illustrates the TABS divisions as: 7.1 In-Situ Resource Utilization; 7.2 Sustainability and Supportability; 7.3 Advanced Human Mobility Systems; 7.4 Advanced Habitat Systems; 7.5 Mission Operations and Safety; and 7.6 Cross-Cutting Systems.

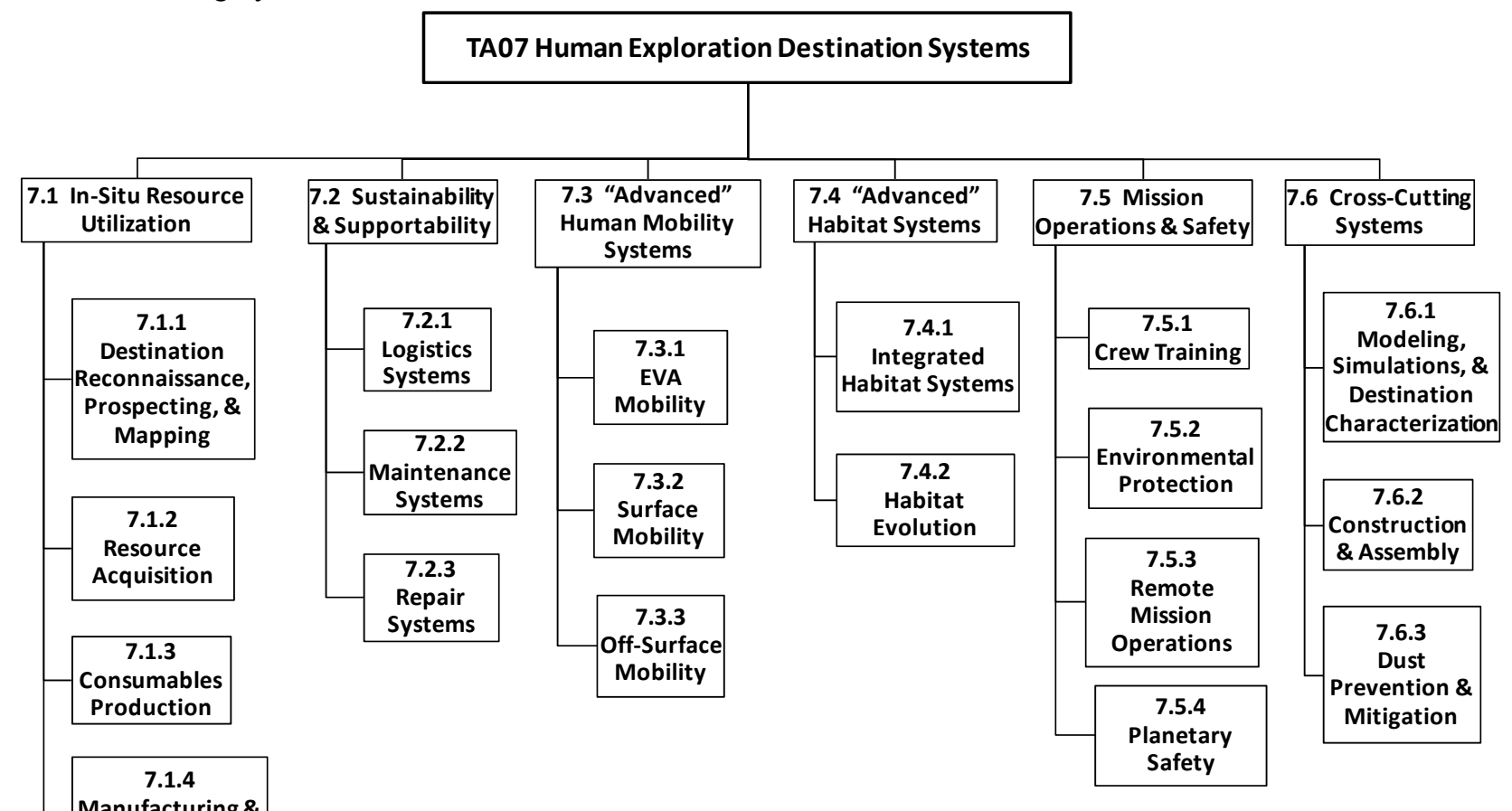

Figure 2: TA7 HEDS Technology Area Breakdown Structure

The HEDS TABS was used to map the respective fundamental and applied technologies to the four NASA Mission Directorates' pull missions as applicable (fig. 3). The applicable "pull-mission" is shown at the top of the roadmaps indicated as a triangle $(\mathbf{\Lambda})$ symbol. Each of the Mission Directorates (Space Operations Mission Directorate (SOMD), Exploration Systems Mission Directorate (ESMD), Science Mission Directorate (SMD), and Aeronautics Research Mission Directorate (ARMD)) is identified by their respective color. "Push" mission opportunities are identified by the HEDS Team's Mission Capabilities Milestones "swim lane", and technology areas are indicated by a star ( $)$ symbol on the HEDS roadmaps shown in figure 3. The HEDS milestones shown on the roadmaps indicate the timeframe that the hardware and/or software should mature to a TRL of 6 . This indicated timeframe is based upon assuming a mission Preliminary Design Review (PDR) occurs at launch minus four years (L-4) for robotic missions and launch minus six years (L-6) for human missions. Thus, if a human mission identified by a Mission Directorate is 2020, then the technology needs to be matured to TRL6 by 2014 . 


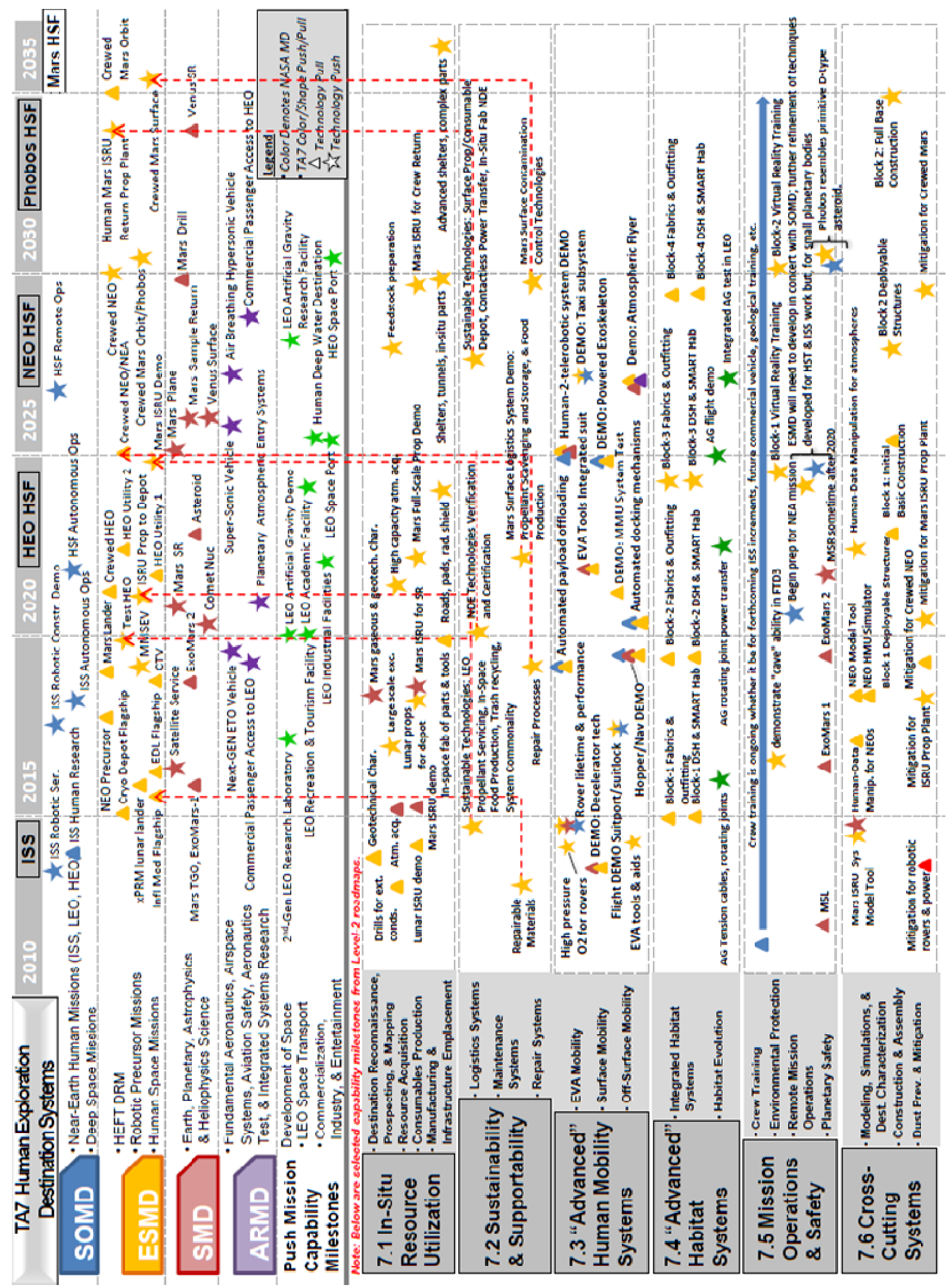

Figure 3: Human Exploration Destination Systems Level-1 Technology Area Strategic Roadmap 


\section{General Overview}

The HEDS technology area is unique from other technology areas since it is a compilation of fundamental and applied technologies for human exploration destinations and is quite diverse and expansive. The HEDS team has strategically road-mapped technology investments that will enable sustained human exploration and support NASA's missions and goals for the next 20-30 years. The HEDS team identified and quantified technological challenges and investments that will be required to safely and cost effectively enable human exploration missions of discovery for our nation, the planet, and for the benefit of all mankind. The following report sections clarify the TA07 technical approach and process that was used to define the TA07 HEDS roadmap.

\section{A. NASA Office of Chief Technology Approach}

HEDS is responsible for understanding NASA's stakeholder capability needs (see Table 1) and diverse challenges that will need to be overcome. A high-level assessment of current technologies capable of meeting these challenges was investigated and a gap analysis performed. Where capabilities did not meet needs, recommendations were provided for investing in technology developments according to anticipated return on the investment. The HEDS team formulated technology investment strategies by outlining technology development concepts and then aligning these to the roadmap milestones. This TA07 HEDS strategic roadmap is a "living document" that is expected to be updated and maintained as designated by the OCT.

Table 1: Sustained Human Presence Needs

\begin{tabular}{|l|l|}
\hline \multicolumn{1}{|c|}{ HEDS Technologies Enable } & \multicolumn{1}{|c|}{ Destination Systems Capability Needs } \\
\hline - Sustained human presence in space & - Launch \& Propulsion Systems (TA01, TA02, TA09, TA13) \\
including planetary bodies & - Habitat Systems (TA06, TA07, TA12) \\
- Remote Earth analog destinations and & - Laboratory Systems (Geo-Sciences, Physical Sciences, Life \\
research & Sciences) (TA06, TA07, TA08) \\
- Commercialization of LEO destinations & - Mobility Systems (TA04, TA07) \\
- Development of GEO & - Autonomous \& Intelligent Operations (TA04, TA07, TA11) \\
$\begin{array}{l}\text { - In LaGrange point destinations } \\
\text { (spacecraft) to destinations }\end{array}$ & - Power Generation \& Stowage (TA03) \\
- Exploration of NEO destinations & - Thermunication Systems (TA05) \\
- Exploration of surface destinations & - ISRU Systems (TA07) \\
& - Logistics Systems (TA07) \\
& - Manufacturing \& Repair Systems (TA07, TA10, TA11, TA12) \\
& - Aio-Technology Systems (TA06, TA07, TA10) \\
& - Medical Systems (TA06, TA08) \\
\hline
\end{tabular}

\section{B. HEDS Technical Approach}

The HEDS core team of 6 was selected by NASA HQ (fig. 4). The technical approach was first to define the HEDS vision, mission, goals and objectives for this technology area. The team looked at multiple human exploration destinations to determine the capabilities and performance that would be required to enable selfsustaining exploration to these destinations. This guided the team through technology brainstorming and identification of evolutionary and revolutionary technologies.

The process used to develop the space technology capabilities roadmaps began with defining the scope of HEDS. The TA07 team focused on identification of drivers, such as "Pull" capabilities and its subsequent technologies from the NASA Human Spaceflight Architecture design reference missions (DRM); Human Exploration Framework Team (HEFT), NRC Decadal Surveys, etc. The team also identified drivers that "Push" capabilities and its subsequent technologies by reviewing past capabilities and game changing technology reports, with a focus on lowering mission cost, and increasing mission autonomy. The TA07 team meet with each of the NASA Mission Directorates customers to map their needs and long-term plans against the preliminary TA7 breakdown structure (table 2). Once this was completed, the team defined top level HEDS technology challenges and then developed first order WBS sub-elements, assigning team leads for each sub-element. The team then defined technology challenges for each sub-element and further decomposed each sub-element into level-3 and level-4 specific technologies required to develop the high level capabilities. The team then generated capability driven roadmaps, assuming no funding constraints and including demonstration flights prior to operational use. Previously developed 
and subject matter experts were consulted to determine technology development timelines. The next step in the roadmap definition process was to define dependencies and collaborations with the other 13 technology areas. This was performed by using a matrix to highlight the dependencies in a quick look graphic and detailed descriptions of the technology dependencies. A similar process was performed to identify the TA7 benefits to national needs. All of this was then documented in a 30-page limit draft report provided to NASA for internal review using the Report Item Discrepancies (RID) review process. RID owners were contacted pertaining to the content of their RID and then dispositioned as accepted, accept with modifications, or rejected. The draft report was updated and submitted to the National Research Council for an independent review.

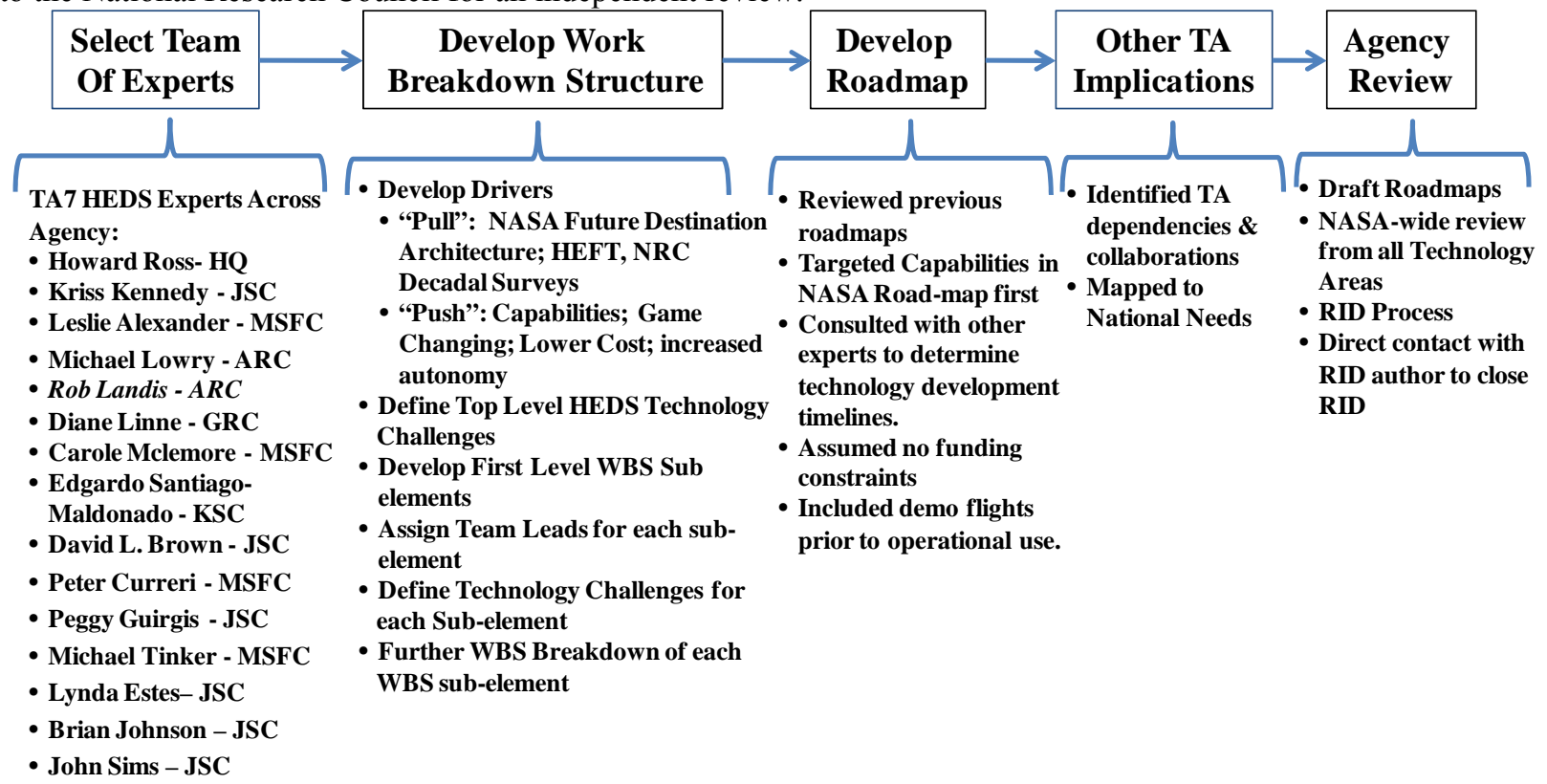

Figure 4: TA07 Roadmap Process

Table 2: HEDS TA Mission Directorate Interests

\begin{tabular}{|c|c|c|c|c|c|}
\hline \multicolumn{2}{|c|}{ TA7: Human Exploration Destination Systems } & SMD & SOMD & ARMD & ESMD \\
\hline $\begin{array}{l}7.1 \text { In-Situ Resource } \\
\text { Utilization }\end{array}$ & $\begin{array}{l}\text { This area covers the identification, acquisition, and } \\
\text { utilization of in situ resources including natural and } \\
\text { man-made. }\end{array}$ & $\mathbf{x}$ & $\mathbf{x}$ & $\mathbf{x}$ & $\mathbf{X}$ \\
\hline $\begin{array}{l}7.2 \text { Sustainability \& } \\
\text { Supportability }\end{array}$ & $\begin{array}{l}\text { This area covers operations and the sustainability of } \\
\text { humans and required vehicles and systems. }\end{array}$ & $\mathbf{x}$ & $\mathbf{x}$ & $\mathbf{X}$ & $\mathbf{X}$ \\
\hline $\begin{array}{l}\text { 7.3 Advanced Human } \\
\text { Mobility Systems }\end{array}$ & $\begin{array}{l}\text { This area covers the transportation or mobility of } \\
\text { humans and cargo in the near proximity of the } \\
\text { destination or on the surface. }\end{array}$ & & $\mathbf{X}$ & $\mathbf{X}$ & $\mathbf{x}$ \\
\hline $\begin{array}{l}\text { 7.4 Advanced Habitat } \\
\text { Systems }\end{array}$ & $\begin{array}{l}\text { This area covers advanced habitat technologies of } \\
\text { deep space habitat capabilities that enable long- } \\
\text { duration and deep-space human missions. }\end{array}$ & & $\mathbf{X}$ & & $\mathbf{x}$ \\
\hline $\begin{array}{l}7.5 \text { Mission } \\
\text { Operations \& Safety }\end{array}$ & $\begin{array}{l}\text { This area covers the safety and health of humans, } \\
\text { vehicles, systems, and destinations. }\end{array}$ & $\mathbf{x}$ & $\mathbf{x}$ & $\mathbf{X}$ & $\mathbf{x}$ \\
\hline $\begin{array}{l}7.6 \text { Cross-Cutting } \\
\text { Systems }\end{array}$ & $\begin{array}{l}\text { This area covers the identification and } \\
\text { characterization of destination targets, systems } \\
\text { engineering, and technologies for construction, } \\
\text { assembly, and deployment of destination systems } \\
\text { hardware. }\end{array}$ & $\mathbf{x}$ & $\mathbf{x}$ & $\mathbf{x}$ & $\mathbf{x}$ \\
\hline
\end{tabular}




\section{Current State of the HEDS Technology}

As a space agency, NASA has developed a number of technology roadmaps over the past 10 years, notably Stepping Stones (2001); THREADS (2002); Capabilities Requirements, Analysis, and Integration (CRAI, 2004); Advance Planning and Integration Office's (APIO) Strategic and Capabilities Roadmap (2005); and Technology Horizons (2009). These roadmaps were reviewed and data mined for pertinent HEDS technologies.

Most of the HEDS evolutionary technologies are at a Technology Readiness Level of 3 or 4 (TRL-3 to TRL-4). Funding for technology development has been sporadic over the years absent of a focused effort that is needed to take the technologies from the bench-top component level to integrated systems level testing in a relevant environment. Technology maturation to TRL-6 is required prior to the element or system Preliminary Design Review (PDR). Once a technology has matured to TRL-6, responsibility will be transitioned to the element or system project for flight hardware development. Revolutionary technologies identified by HEDS are as "push" capabilities are typically at TRL-1 or TRL-2. For example there needs to be basic and fundamental technology development in micro-sensors, nano-technologies, bio-technologies, and advanced "smart" materials before they can be integrated into an applied technologies development program.

True 'game-changing' technologies and capabilities need to be developed and demonstrated to a high TRL even earlier than the mission PDR according to their role in a mission. For example, if in-situ produced propellants will be used to return crew from Mars, there must be a high level of confidence in this game-changing, but high-risk, technology in order to be included in the critical path at the onset, which may occur $10-15$ years before flight. It is proposed that these technologies may need to have full-scale demonstrations in the environment by the mission planning stage. Again looking at Mars ISRU as an example that may mean sending a full-scale plant to Mars at least 10 years before launch of the human mission to demonstrate the ability to produce, store, and transfer propellants necessary for human Mars return flight.

\section{HEDS Benefits}

The technologies included in the HEDS roadmap will provide numerous benefits to the various exploration missions planned. Some of these benefits are discussed here.

Revolutionizes Architectures and Mission Planning: The cost and effort to escape the Earth's gravity well is the overwhelming factor limiting our exploration of space. Technologies such as in-situ production of propellants, in-space construction and assembly, deployable structures, integrated logistics, and automated maintenance and repair will sever the anchor to the Earth supply chain and lead the way to permanent human presence.

Guides Identification of Human Accessible Targets: To date, we have only discovered about 3 percent of the known NEO population. A NEO survey mission such as a space-based infrared (IR) survey telescope in a Venuslike orbit about the Sun will provide characterization data and perform foundational solar system science of NEOs, comet families and Main Belt asteroids. Such a mission will reveal both new planetary targets for robotic and human exploration and all potentially hazardous bodies that may collide with Earth and has implications for U.S. National Security and ultimately the survival of the human species.

Increases Crew Productivity and Science Return: Due to the complexity of spacecraft systems and inherent remoteness of any space mission, crews spend much of their time performing routine health monitoring, system maintenance, and repair. Technologies such as robotic maintenance and repair, self-diagnostics, verification, and reconfiguration, and 'smart' self-healing habitation structures and other self-repair components can greatly reduce time spent on general housekeeping and safety activities and free up the crew for science and exploration. Crew training time and costs will also be significantly reduced. Mobility technologies such as more adaptive EVA spacesuits, suitports and/or suitlocks, exoskeletons, rovers, hoppers, surface transports, and atmospheric flyers and/or near-surface manned maneuvering units will increase the translation range for the crew and significantly increase the science return for every mission.

Reduces Launch and Mission Costs and Increase Crew Safety: The cost of launching logistics and supplies from Earth is a major factor in overall mission cost. Deep space human missions will require large amounts of mass for consumables and spare parts. An integrated logistics system that provides a centralized depot managing consumables produced in-situ from local resources and recycled resources brought from Earth or found at the destination will reduce or eliminate this supply chain. Technologies such as consolidation of similar systems, insitu automated maintenance and repair, reuse and recycling of hardware and residuals, and preservation and production of food for in-transit space and destinations will reduce overall mission cost, increase crew safety, and increase scientific mass allocation for instruments.

Enables Commercial Spin-Offs: Many of the HEDS technologies have great potential and value to commercial industries for future technology spin-offs and developments which can be licensed under technology transfer 
agreements. These in turn can stimulate new industries, job growth, and new exciting educational opportunities advancing the United States' leadership in technology developments.

Cultivates Commercialization and Development of LEO: With the exception of a handful of multi-millionaire paying 'astronauts', access to space has been limited to government space agency crews. Technologies that enable integrated habitat systems such as advanced fabric materials, light-weight windows, 'smart' Hab systems, sensors and healing, deployable structures, and artificial gravity capabilities for deep-space missions can also be applied to commercial structures in LEO. Development and demonstration of in-situ technologies such as production of propellants and consumables and in-situ manufacturing could also contribute to this exciting new market. This would open up the experience of space for generations to come by exciting and engaging the public and taxpayers.

Promotes STEM Careers: It is the human element in space exploration that provides the incentive and excitement for young people to choose to pursue space flight and all of its challenges and opportunities. This innate desire to explore our universe and beyond requires the proper preparation and education of the future workforce. All of the technologies described in the HEDS TA that will enable exciting and continued human space flight to deep-space will also inspire the next generations to enter the science, technology, engineering and mathematic (STEM) fields in order to continue our legacy and the pursuit of knowledge through exploration.

\section{E. Applicability/Traceability to NASA Strategic Goals}

The "NATIONAL SPACE POLICY of the UNITED STATES of AMERICA" identifies six (6) strategic goals for the national space program that the HEDS area addresses. The HEDS technology area contributes to meeting five (if not all six) of the goals listed below.

- Energize competitive domestic industries to participate in global markets and advance the development of: satellite manufacturing; satellite-based services; space launch; terrestrial applications; and increased entrepreneurship.

- Expand international cooperation on mutually beneficial space activities to: broaden and extend the benefits of space; further the peaceful use of space; and enhance collection and partnership in sharing of space-derived information.

- Strengthen stability in space through: domestic and international measures to promote safe and responsible operations in space; improved information collection and sharing for space object collision avoidance; protection of critical space systems and supporting infrastructures, with special attention to the critical interdependence of space and information systems; and strengthening measures to mitigate orbital debris.

- Increase assurance and resilience of mission-essential functions enabled by commercial, civil, scientific, and national security spacecraft and supporting infrastructure against disruption, degradation, and destruction, whether from environmental, mechanical, electronic, or hostile causes.

- Pursue human and robotic initiatives to develop innovative technologies, foster new industries, strengthen international partnerships, inspire our Nation and the world, increase humanity's understanding of the Earth, enhance scientific discovery, and explore our solar system and the universe beyond.

- Improve space-based Earth and solar observation capabilities needed to conduct science, forecast terrestrial and near-Earth space weather, monitor climate and global change, manage natural resources, and support disaster response and recovery.

\section{F. HEDS Top Technical Challenges in Chronological Order}

Each technology area team was asked to identify their top ten technology challenges. For the HEDS TA that covered such a wide range of technologies, this proved to be a difficult challenge. Below are the top (more than ten) challenges identified by the team in chronological order. It should be noted that the timeframe listed is when the technology needs to reach TRL-6; work on many of these top challenges will need to be started in an earlier timeframe to meet the need dates indicated.

2010-2016:

- Suitports, suitlocks, \& alternative airlock systems: A review of current space station procedures reveals Pre EVA time of 158 minutes, depress 30 minutes, and post EVA time of 80 minutes. Implementing an alternative airlock approach that also does not require the full crew would reduce the ops time to prepare for and depress from EVA significantly (given crew saturation at reduced pressure), allowing more time for crew exploration. Design must be adaptable to multiple crew members. Technologies include mechanisms and seals 
that are dust tolerant or resistant in harsh environments for extended lifetimes, integrated quick disconnect umbilicals and sensors for supply gasses, lightweight, dust resistant materials, efficient air recovery pumps, and manufacturing techniques

- Advanced materials and processes to enable in-situ repair: The in-situ and self repair capability will increase human exploration safety and will decrease spare parts mass. Advanced self-repair and self-diagnostic materials will have the capability to repair as damage occurs and will provide early notification of the existence of damage before failure. This would allow a pro-active approach to maintenance and repair, versus the current reactive approach. Advanced repair processes will allow for the in-situ repair of damaged components. These processes will minimize the spare parts manifest increasing scientific payload capability or decreasing overall launch mass.

- Development of crew autonomous and spacecraft autonomy concepts: NASA currently employs a number of reliability tools and methods, including Failure Modes and Effects Analysis (FMEA), Fault Traceability Analysis (FTA), and Probability Risk Analysis (PRA), and design engineers have used them successfully for designing reliable and safe systems. But these methods have drawbacks that limit their applicability to early phase design and design for ISHM. As we study the particular goals for ISHM design, we can select and extend risk and reliability methods to suit those goals. We have already begun developing failure analysis methods that determine failure modes during the early stages of functional design. It is essential to begin tools for early stage design (even conceptual design) because the early stage affords the best opportunity to effectively catch and prevent potential anomalies and failures.

- Resource Acquisition Technologies: While people have been digging on Earth for hundreds of years, excavation on planetary bodies requires low mass, low power, and highly robust equipment, while excavation time constraints are typically much relaxed - all uniquely different than terrestrial excavation requirements. In addition, granular flow on Earth is not a well-defined technology and solutions typically involve human intervention (e.g., kick the machine when it clogs). Granular flow in unique vacuum environments with highly irregular shaped particles will be even more challenging. There is no terrestrial precedent for acquisition of large amounts of very low-pressure gas as needed to initiate in-situ resource utilization on Mars.

2017-2022:

- Radiation protection technologies: The greatest environmental risk to human spaceflight beyond the Earth's magnetosphere is radiation. Ionizing radiation, galactic cosmic radiation (GCR), and solar cosmic radiation (SCR), is a significant risk to astronauts for all long-duration missions beyond LEO regardless of destination (NEO, lunar surface, or Mars). Many of these high energy elementary particles penetrate spacecraft, pressure vessels and space suits (generating secondary radiation particles in the process) as well as organs, cells and DNA of human occupants inducing degenerative changes usually associated with accelerated aging (extracellular matrix remodeling, persistent inflammation, oxidative damage, cataracts and damage to the central nervous system). DNA damage results in increased mutation rates, genomic instability, cancer induction and activation of latent tumors. Effective strategies for mitigating space radiation hazards must be developed, tested, and verified if mission success is to be assured. Technologies for shielding approaches such as placement of equipment, food, water and waste material in long-duration spacecraft; polyethylene-lined sleep stations or internal 'shelters;' external shielding approaches such as storable propellant placement, use of NEO regolith 'sandbagging' to protect crew compartment(s) during proximity ops and return phases of the mission, radioprotective pharmaceuticals (TA06) that could be taken as a preventive or in response to a significant increase in radiation (such as an SPE) and plastics/polymers need to be developed and tested

- Technologies to minimize mass of cargo and logistic needs from Earth: Early demonstration of these highrisk high-payoff technologies will provide the needed maturity level of the technology in time to be inserted into the critical path of human space exploration mission architectures. An integrated logistic depot to store, recycle, and distribute consumables will reduce significant system mass by consolidating redundant subsystems (e.g., centralized electrolysis and fuel cell systems, consumable liquefaction and storage, etc). Trash processing into consumables will provide useful consumables while reducing waste accumulation during long transit time and provide a trash management technique while at the destination.

- Advances in "smart" Habs Technologies: Current ISS modules are Mission Ops labor intensive and require constant crew interaction. Advanced "smart" Habs will enable autonomous operations of habitats for predeployment and while the crew is using them. This will enable the crew to perform valuable exploration 
objectives pertaining to scientific discoveries and knowledge capture. A DSH will need this technology capability thus decreasing response time in an emergency situation and thus increasing crew safety. Technologies include ( smart homes/buildings) integrated software controls, self-repairing, automated lighting, communications, \& systems control, person recognition, micro-systems integration, patching \& repairing, manufacturing technologies.

- Advances and increases in crew autonomy: There are several situations where crew autonomy is essential to mission success: when the time-to-criticality (TTC) of ANY situation, not just a system fault, is less than the time needed to communicate with mission control; when there is not a pre-planned option to respond to a contingency - crew must contact the ground (for support and to analyze) - determine the best method to proceed and then continue with the recommended action; when a fault results in the loss of communications, then the situation is intensified because the crew cannot even notify MCC of the problem. Autonomy and autonomous systems not only enhance deep space human expeditions, but enable such voyages. This is first and foremost a safety concern. The distance and complexity of such missions drive the need for autonomy. And, as systems become more complex, particularly for multiple vehicles and elements, the number of permutations in fault modes increases exponentially.

- Advances in integrated Hab shell technologies: Current ISS modules are aluminum skinned with outer layers of protection for MMOD, thermal, atomic oxygen, UV, etc. This individualized approach adds additional weight, inefficiencies in protection systems and mass optimization. Advanced integrated shells can optimize between primary pressure vessel, protection systems, and integration of utilities such as thermal control, power distribution and advanced materials. Technologies include integrated radiation \& environmental protection, integrated power systems, integrated thermal systems, integrated communications antenna systems, nano technologies, integrated sensors, advanced materials, and bio-technologies.

- Solids and fluids processing technologies: Many different technologies for processing resources and extracting useful products have been identified, and several have been demonstrated to various levels. A concerted effort is needed to fully understand each process to identify best candidates and optimize the entire system.

2023-2028:

- Technologies to enable reconfigurability and reusability of components and sub-systems: The ability to repair complex systems requires that systems be designed to allow for minimum intrusive repairs as well as for reusability of critical components. System Reconfigurability and Reusability requires technologies (software and hardware) to reconfigure integrated systems to isolate the area that requires repair without affecting the availability and operation of the entire system. Reusability and redundancy of mission critical hardware is a needed technology to allow for non-critical components and systems to be used to repair or to replace failed mission critical components or systems. Technologies include enabling maintainable and repairable systems: maintenance real-time reconfigurable software, reprogrammable avionics, and reconfigurable systems.

- Large scale dust removal and mitigation: Once we start planning for longer duration human exploration on planetary surfaces such as Mars or the moon, we will need methods to prevent and remove dust from critical surfaces in order to extend the life of the surface systems. Technologies include dust removal from large (habitat) surfaces and windows, electrodynamic repulsion for large surfaces, electron discharge automated cleaning system, improved suit cleansing in airlock before entering $\mathrm{Hab}$, and $\mathrm{CO}_{2}$ snow showers to improve efficiency of electrodynamic removal of fine dust from suits.

- In situ manufacturing and infrastructure technologies: Every exploration plan calls for sustainable exploration architectures. Consumable production is the first step in breaking the Earth logistics supply chain, but explorers will not experience complete sustainability and self-reliance without the ability to create structures and manufacture spare parts and new components at the destination. Technologies include creation of powdered metals from extracted molten metal, additive and 3-D printing fabrication techniques, excavation, movement, and placement of large amounts of regolith, and regolith manipulation \& solidification technologies.

\section{G. HEDS Technology Development Capability Needs}

The HEDS technology area identified several key capabilities needed for the successful development and maturation of human exploration destination systems technologies. NEO survey and characterization missions are required in order to scientifically and engineering-wise understand these NEOs so that the appropriate technologies 
are developed. While several thousands of NEOs have been discovered, it represents only $\sim 3 \%$ of the total known population. Due to their long synodic periods, many may linger on the opposite side of the Sun as viewed from the Earth; while others, interior to Earth's orbit about the Sun are missed entirely. The remaining NEOs could be identified and classified (to first order) with the appropriately instrumented infrared (IR) telescope in a Venus-like orbit. Such a telescope will reveal potential new targets to enable human sprint missions (i.e., $\sim 150$ days or less) into the solar system. These newly discovered NEOs will be of high interest for human missions for their relative accessibility (in terms of total Dv), their potential resources $\left(\mathrm{H}_{2} \mathrm{O}\right.$, mineralogical wealth, etc.), and a definitive feedforward to the vicinity of Mars. Another capability needed is a "dirty" thermal vacuum chamber to simulate NEO or planetary surface environments and test hardware systems and components that will interact with the highlyabrasive regoliths. It is imperative these tests occur in this type of simulated environment at vacuum and extreme temperatures. High quality NEO and planetary simulants are required for component, sub-system, and system-level tests to ensure hardware will operate as expected in the actual environment.

Several push missions were identified that moved up technology need dates in several elements. One of these push missions is to supply the existing propellant depot (2018 pull mission) with propellants derived from lunar regolith. Studies have repeatedly shown an overall mass-payback for lunar supplied propellants compared to Earthshipped propellants. A second push mission added is a full-scale Mars ISRU demo in 2025. As mentioned earlier, this team feels that substantial technology demonstrations of high-risk, game-changing technologies will be required in advance of mission architecture initiation, which occurs $10-15$ years prior to the mission. The need date for most of the lunar and Mars ISRU technologies are tied to PDR dates for these two push missions.

\section{Detailed Portfolio Discussion}

The HEDS Technology Area Breakdown Structure (TABS) is divided into six Level 2 technology focused areas (figure 2) 7.1 In-Situ Resource Utilization; 7.2 Sustainability and Supportability; 7.3 Advanced Human Mobility Systems; 7.4 Advanced Habitat Systems; 7.5 Mission Operations and Safety; and 7.6 Cross-Cutting Systems. Each Level-2 technology focused area is further broken down to provide additional detail on the content. The following section provides definitions and technology descriptions for the TA07 HEDS Level 2 and 3. Each Level 3 was further decomposed into two additional levels during team brainstorming sessions to provide clarity for creation of the roadmaps.

\section{A. In-Situ Resource Utilization (ISRU) (HEDS TABS 7.1)}

Specifically, ISRU is defined as the identification, acquisition, and utilization of in situ resources whether they are naturally occurring or man-made. The purpose of ISRU is to enable the capability of self-sufficiency at particular locations, especially for destinations far from Earth, thereby eliminating the requirement to launch large amounts of mass for consumables and other items such as structures in order to survive and successfully conduct mission objectives. The ISRU WBS is broken down into four areas as follows: 7.1.1 Destination Reconnaissance, Prospecting, and Mapping; 7.1.2 Resource Acquisition; 7.1.3 Consumable Production; and 7.1.4 Manufacturing and Infrastructure Emplacement (Fig. 5). Each of these areas will be discussed in more detail below. The ISRU Level 2 roadmap shows the detailed fundamental and applied technologies mapped to the pull and push Mission Directorate missions (Fig. 6).

The HEDS TA team has identified several "pull" and "push" missions that would significantly increase the TRL and confidence of ISRU-related technologies that could establish an affordable human space exploration program. It is imperative that these capabilities and technologies be advanced to a TRL 6 before critical path events such as architectures and hardware PDRs occur; otherwise, the risks may be too great to the missions to realize the ISRU benefits in a timely manner. Some of the potential missions currently identified to demonstrate and prove out various ISRU technologies are: (1) an exploration robotic precursor mission to the Moon (xPRM Lunar Lander) in 2016 or as soon as possible in order to better determine lunar geotechnical properties and to demonstrate on a small scale the extraction of water or other materials which will then be used to demonstrate production of propellants on the lunar surface using in-situ resources; (2) a small-scale fabrication/manufacturing demonstration on the ISS in 2017 and the Crewed NEO/NEA in 2025 to make small spare parts or tools using metal materials; 3) an ISRU resource identification and extraction demonstration (small-scale) using Mars regolith on the ExoMar-2 mission in 2017 or the Mars 2018 lander; 4) an autonomous lunar ISRU propellant production system that would provide propellant to the on-orbit propellant depot by $2022 ; 5)$ an exploration robotic precursor mission to Mars to demonstrate full-scale ISRU on Mars using Martian resources by 2025; and 6) deployment of full-scale ISRU propellant production system to Mars by 2034 in support of a crewed mission to Mars by 2036. The following 
HEDS TA Level 3 descriptions address technologies required to meet these missions and other potential human space exploration missions that may be forthcoming.

\section{Destination Reconnaissance, Prospecting \& Mapping (HEDS TABS 7.1.1)}

Before a destination should be aggressively pursued for exploration, the first order of business should be to better understand it. In the case of ISRU, the regolith and any atmosphere or environment should be properly investigated and mapped out for future mining and utilization of mission- and life-sustaining resources at the various destinations including mineral/chemical compositions, quantities, etc. The tasks necessary to perform this element are split as follows: 7.1.1.1 Destination Imaging \& Characterization; 7.1.1.2 Destination Analysis; and 7.1.1.3 Sample Collection and Characterization (Fig. 5). The HEDS TA7 Team coordinated with the Science Instruments, Observatories, and Sensor Systems TA8 Team and came to agreement that the TA08 Team would cover sensors and instrumentation that the HEDS capabilities and technologies would need in order to perform reconnaissance, prospecting, and mapping; thus, those items will not be covered here. After determinations are made as to whether to pursue a destination, then actual samples should be collected and further characterized and analyzed to finalize destination decisions and to perform ground truth studies. Technologies needed for this capability include: penetrometers; shear gauges; compaction/density/flow instruments such as worms, scoops, and drills; and coring drills for purposes of collecting small samples and characterizing them in terms of geotechnical/physical properties, mineralogy/chemistry, dust sensitivities, hazardous materials, etc. As technology advancements proceed in this element, further information such as compaction and traction regarding the regolith behavior while driving or moving across it will be collected. Electromagnetic data (i.e., dielectric properties) will be measured to understand the potential for later using microwaves or other sources for heating the regolith to bind the particles together to construct landing pads or roads or to extract resources such as water or other materials. The collection and characterization of any atmospheric gasses or dust is also vital for understanding the potential for ISRU applications. The capability to perform better prospecting, mapping, and characterization of samples not only increases NASA's returns on exploration investments, it could also revolutionize mining, purification systems, the pharmaceutical industry, and other commercial industries that utilize these types of technologies.

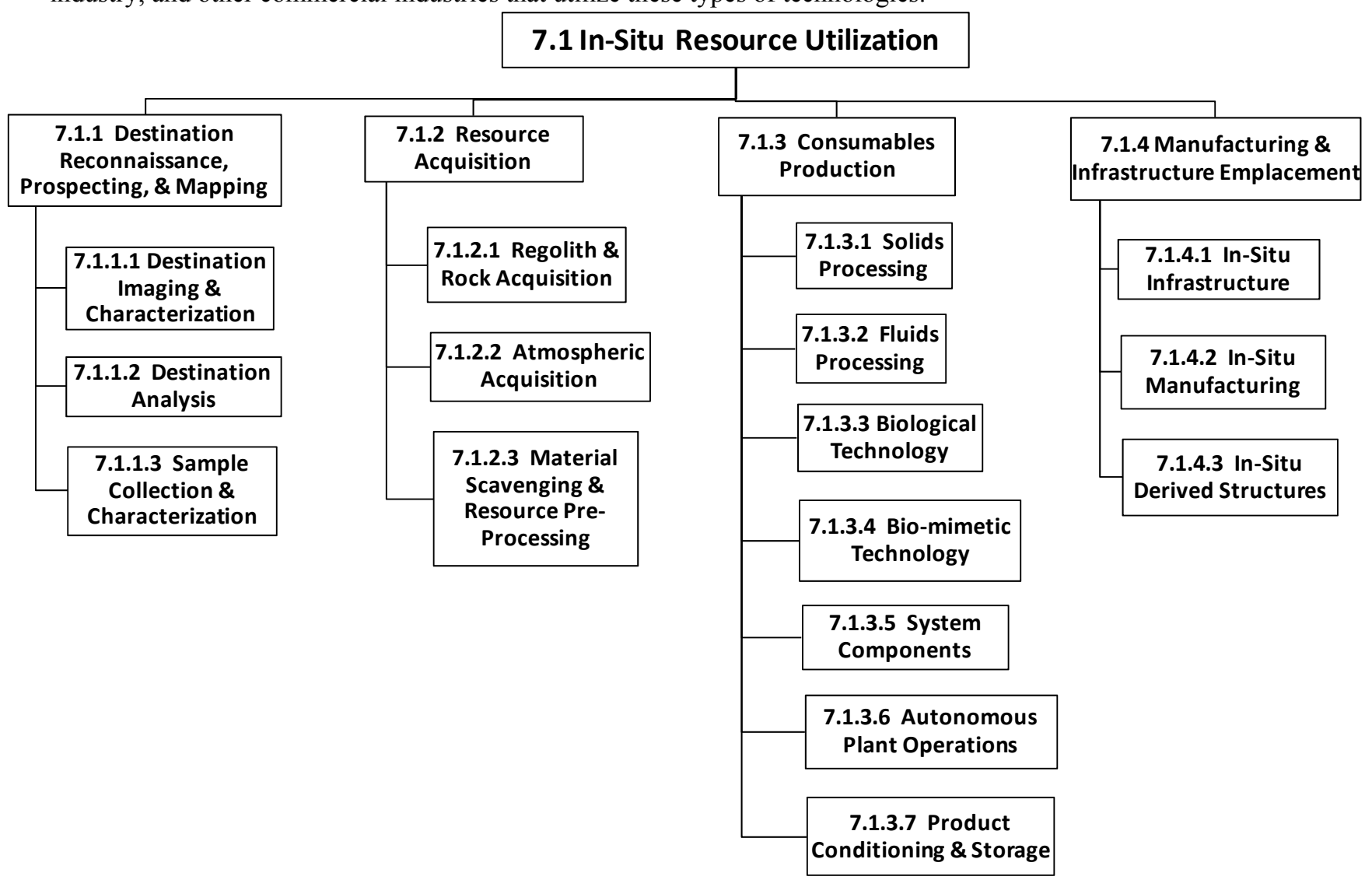

Figure 5: HEDS In-Situ Resource Utilization Level-2 TABS 


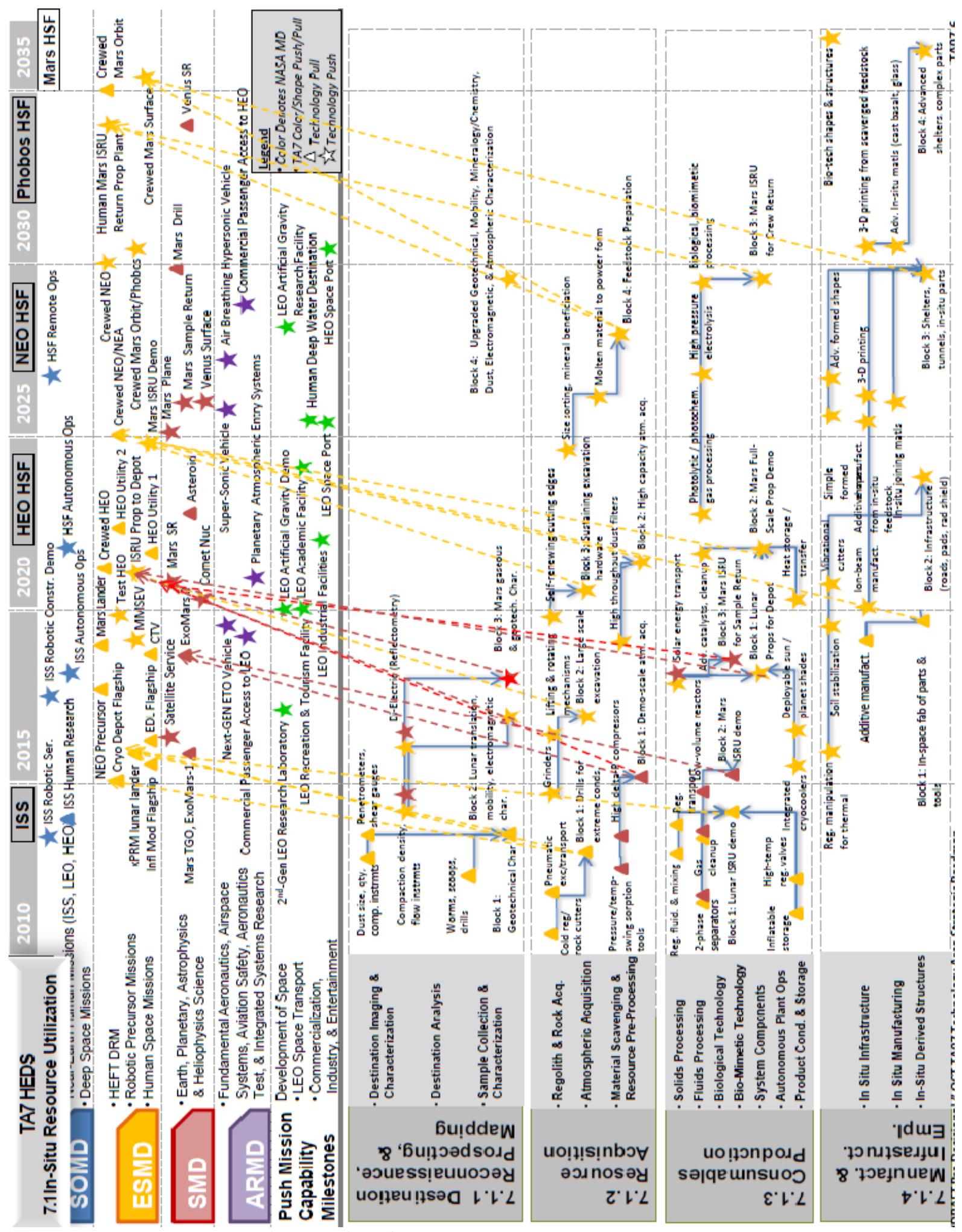

Figure 6: HEDS In-Situ Resource Utilization Level-2 Roadmap 


\section{Resource Acquisition (HEDS TABS 7.1.2)}

This ISRU element pertains to the collecting, extracting, recycling, processing, and storing of targeted "raw" in situ resources as optimal locations have been narrowed down and even pin-pointed based upon the technologies described above. These resources are collected from the following sources (numbers coincide with the WBS): 7.1.2.1 Regolith and Rock Acquisition; 7.1.2.2 Atmospheric Acquisition; and 7.1.2.3 Material Scavenging and Resources Pre-Processing. These raw resources are turned into feedstock that will later be used to produce consumable products that will be discussed in the next section (Fig. 7). Another often overlooked area of importance is the scavenging of material (old or unused/broken hardware, scrap material, consumed propellant or gas tanks, etc.) that could be turned into feedstock for another purpose or product. For example, metal tanks could be crushed and then formed into wire or powder and used in manufacturing processes to make other metal parts.

Multiple technologies are needed for acquiring these various types of resources. These include: special non-clogging, dustresistant cutting tools and drills (including self-sharpening), scoops, lifting and rotating gears, seals, bearings and actuators, and pneumatic excavation and material transport handling equipment, containers, and storage equipment to dig or capture the soil. Equipment to capture the atmosphere such as high delta-pressure and low flow rate compressors, and pressure/temperature extreme sorption tools is required and must be advanced. Pre-existing in-situ hardware such as discarded or used spacecraft can also be scavenged and recycled or re-purposed for "raw" feedstock in preparation for processing. Examples of technologies required for this include crushers and grinders; mineral beneficiation to increase the concentration of desired minerals to be processed; electrostatic separation; size beneficiation; and technologies to process, handle, and store intermediate resources such as molten metal and byproducts. All of these technologies should focus on self-cleaning, non-clogging sieves and filters, gears, bearings, etc.

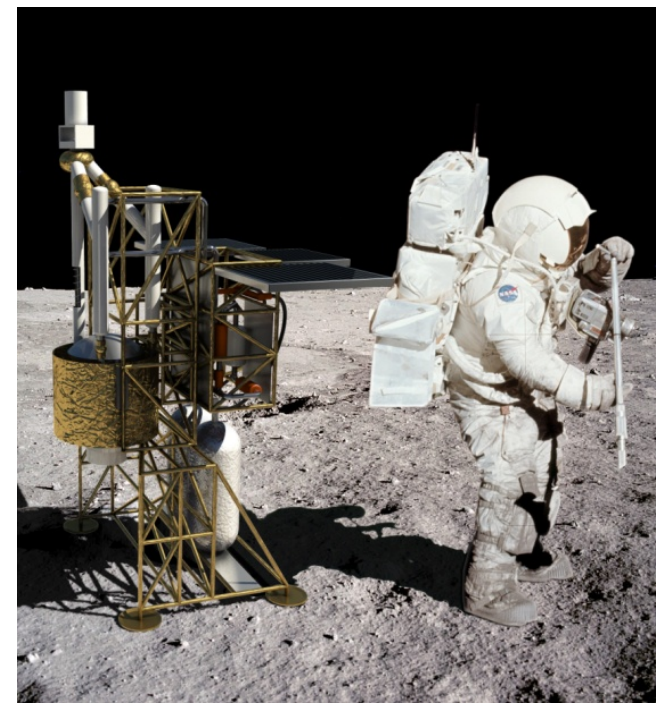

Figure 7: Conceptual Lunar ISRU Processing Plant

\section{Consumable Production (HEDS TABS 7.1.3)}

After the resources are acquired as described above, consumables can be produced from the in situ resources. Examples include consumables such as water, air, food, and propellants as needed by the crew, scientific equipment, robots, rovers, etc. Many different types of production processes are required in order to handle the variety of resource inputs. This ISRU element is delineated as follows: 7.1.3.1 Solids Processing; 7.1.3.2 Fluids Processing; 7.1.3.3 Biological Technology; 7.1.3.4 Bio-Mimetic Technology; 7.1.3.5 System Components; 7.1.3.6 Autonomous Plant Operations; and 7.1.3.7 Product Conditioning and Storage. Technologies that can handle not only solids such as rocks, soil, metals, etc., but also fluids such as gasses $\left(\mathrm{H}_{2}, \mathrm{CH}_{4}, \mathrm{~N}_{2}\right.$, etc.) and liquids $\left(\mathrm{H}_{2} \mathrm{O}\right.$, waste, etc.) must be matured and advanced. Some examples of these technologies include: regolith fluidization and mixing; nonreactive, impermeable, and dust-resistant materials; 2-phase separators; gas cleanup; decontamination; hightemperature chemical reactors (hydrogen reduction, Carbothermal, molten regolith electrolysis, etc.); high pressure, low/high temperature containers; sun/planet shades; multiple levels of containment storage devices; solar/thermal energy concentrators and transport; photolytic/photochemistry gas processing; electrolysis; catalysts; and cryocoolers to name a few. Relatively new to space applications, technologies that address both biology and biomimetic fields are expected to make great strides in producing consumables in situ or transforming toxic or hazardous waste products into "green" products as well and should be addressed.

It is important to develop technologies to address the cross-application of system components such as seals, valves, mechanisms, etc. to reduce the different types of spares as well as decrease the crew training requirements. Technologies that result in autonomously operating in-situ production plants safely and efficiently must be developed and advanced and includes mechanical and thermal components such as: long-life, high-cycle valves, actuators, mechanisms, radiators, heat pipes, bearings, seals, lubricants or (lubricant-free items) etc. that tolerate high-temperatures, high pressures, caustic gases, and/or extreme dirt and dust exposure. Last, but not least, the consumables that are produced as a result of the aforementioned technologies and applications require proper storage and handling in order to ensure the products have not expired and are still safe for consumption and use. 


\section{Manufacturing and Infrastructure Emplacement (HEDS TABS 7.1.4)}

This ISRU section included utilizing resources (metals, plastics, regolith, etc.) acquired from the land or environment in situ to create infrastructure, fabricate tools and parts, and construct items as needed for safety, redundancy, comfort, utility/functionality, etc., thereby, reducing the need to launch all items from Earth. The benefits of having the capability to manufacture or fabricate parts, tools, etc. on-demand in situ include decreased risk to humans and increased mission success. Specifically, this area addresses: 7.1.4.1 In-Situ Infrastructure; 7.1.4.2 In-Situ Manufacturing; and 7.1.4.3 In-Situ Derived Structures. Technologies using microwaves, ionic liquids, solar/thermal energy, binders, adhesives, covers, and excavation equipment are required for preparing roads, berms, landing pads, habitats, garages, and other items that will reduce dust kick-up and contamination, protect spacecraft and humans from blast ejecta and the environment, and provide ease of translation/mobility. Regolith packing technologies for building radiation or solar storm shelters are required as well as technologies for modifying the thermal properties of regolith for thermal management and energy storage and savings. The production of solar cells from in situ material must be demonstrated and eventually developed into a power grid as infrastructure. Other technology advancements that should be advanced include: bio-technologies that can grow habitats (shells) and shelters; materials capable of withstanding different environmental parameters; and low power, deployable, autonomous manufacturing plants.

Extremely critical technologies necessary for survival at far-away locations include those that can manufacture or fabricate spare parts, tools, radiation shields, equipment, and other structures using the regolith (lunar-crete or Marscrete) and/or resources mined from the regolith such as metals, glass, etc. These new manufacturing technologies such as electron beam, 3-D printing, ion beam, machining processes, joining/structural tape, welding, etc. save time (labor) and material by reducing waste, especially in the case of additive manufacturing. Humans cannot wait several months or years until new parts arrive to repair critical systems such as Environmental Control and Life Support Systems, for example. Machines made to self-replicate, self-repair could result in mini-factories that are mobile to go wherever/whenever. The capability to manufacture items will reduce the up-mass required for spares resulting in room for scientific instruments that can be used for true exploration and discovery.

\section{B. Sustainability \& Supportability (HEDS TABS 7.2)}

The Sustainability and Supportability (SnS), TABS 7.2 (figure 8), includes all the technologies required to establish a self-sufficient, sustainable, and affordable human space exploration program. SnS is divided into three major technology areas to tackle the aforementioned characteristics and/or requirements for human space exploration: 7.2.1 Logistics Systems, 7.2.2 Maintenance Systems, and 7.2.3 Repair Systems. Each of these technology areas will be discussed in details in the sections below. Most of the SnS technologies, specifically Maintenance and Repair Systems, are system specific and must be developed in combination with HEDS development and design.

The SnS Level 2 technology roadmap follows three major developmental phases, fig. 9. The near-term developmental phase (present-2017) focused on the basic research and technology demonstration in a small scale and relative environment, as the case of utilizing ISS as a technology testbed facility, to increase the technology readiness level. The mid-term developmental phase (2017-2023) focus on technology development and application of technology developed during the previous phase that is applicable to human mission to LEO and beyond (i.e., crewed missions to HEO and NEO/NEA). The long-term developmental phase (2023-beyond) focus on technology development as it relates to a push mission of crewed-Mars surface mission.

\section{Logistics Systems (HEDS TABS 7.2.1)}

The Logistics System technology area includes technologies needed to institute a centralized logistic depot to manage and optimize the use of consumables at the human exploration destination and to minimize human-specific logistics (i.e. food) as well as other logistics items that can be repurposed or recycled in order to reduce Earth dependency and logistics train. To address these needs the Logistics Systems is broken down into (1) Consumable Storage, Transfer, and Delivery, (2) Food Production and Preservation, and (3) Reuse and Recycle. The Consumable Storage, Transfer, and Distribution technology area cover those technologies required to establish an efficient centralized consumable depot at the human exploration destination. The Food Production and Preservation technology area includes those technologies required for the efficient production and preservation of food during transit and at the specific human exploration destination. The Reuse and Recycle technology area is a combination of technologies as well as a system engineering approach to design human exploration systems and components with a multi-purpose use goal; however, new technology is required to enable multi-purpose system/component designs. 


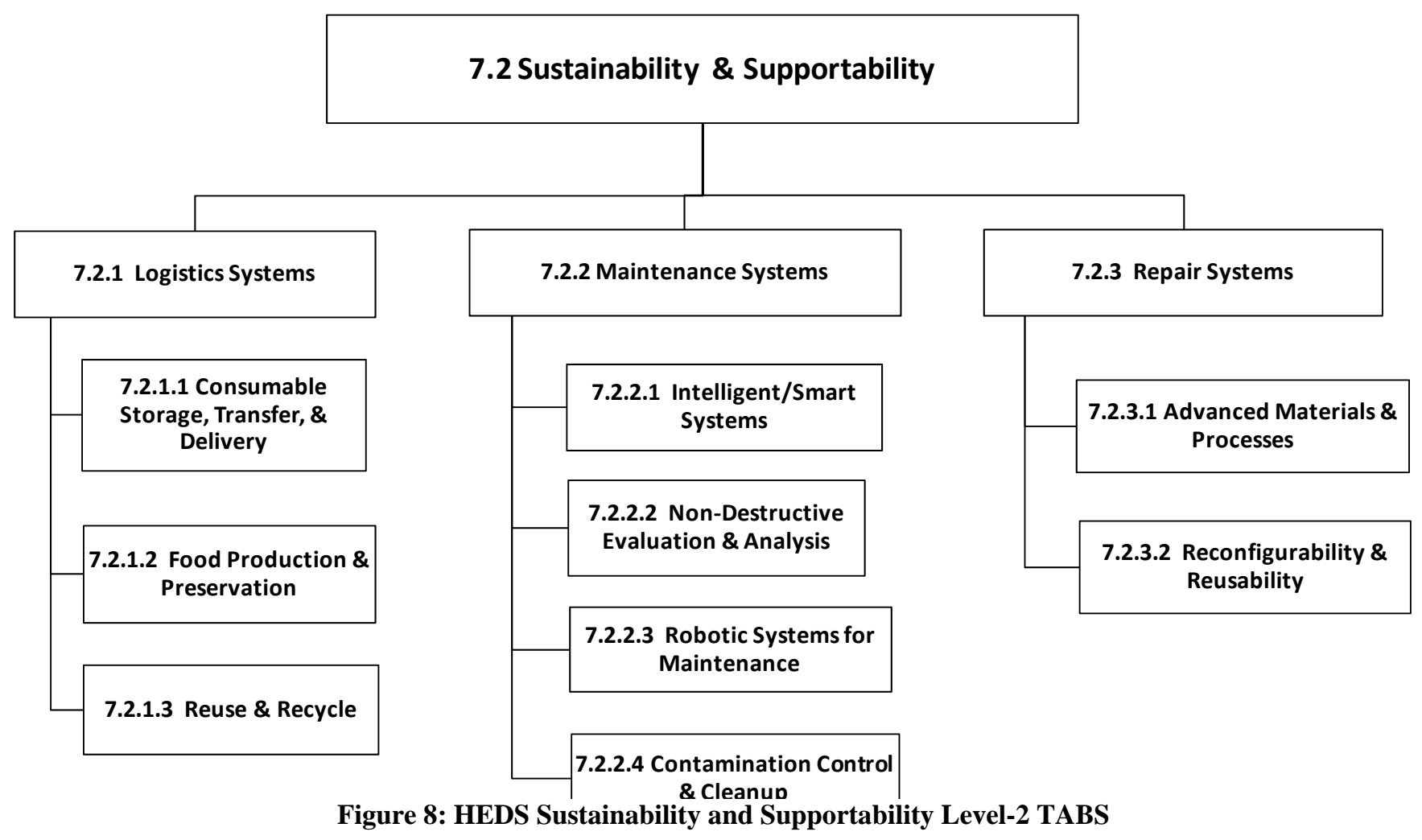

The Consumable Storage, Transfer, and Distribution technology area cover those technologies required to establish an efficient centralized consumable depot at the human exploration destination. The current LEO human space exploration architecture does not make use of a centralized consumable depot as it would be required for deep space human exploration architecture. The centralized consumable depot will include consumables such as propellants, life support consumables, and energy. An efficient consumable depot would require technologies to minimize, if not completely eliminate, the consumable losses; hence, maximizing the use of consumable delivered from Earth as well as those produced in-situ. The technologies needed for a consumable depot include: (a) zero-loss consumable long-term storage and transfer system with autonomous and semi-autonomous operations, (b) low mass and volume storage systems (e.g. cryogenic storage, collapsible storage vessels, etc), (c) autonomous and semiautonomous fluids quick-disconnect (includes dust tolerant), (d) wireless (contactless) power transfer, (e) high pressure gaseous delivery systems, and (f) storage and delivery of other consumables such as glasses, polymers, plastics. Figure 10 depicts a stationary and a mobile consumable depot applicable to surface systems.

The Food Production and Preservation technology area includes those technologies required for the efficient production and preservation of food during transit and at the specific human exploration destination. The in-situ food production and preservation will significantly reduce logistics mass of LEO departure stage, as well as providing an increase to astronauts' psychological and morale from being able to consume fresh food during long travel times to deep space destinations. Technologies required in this area include (a) in-space food production, (b) long-term food preservation (e.g. two or more years for round trip deep space destinations), and (c) in-situ food production at the human destination. These technologies must demonstrate low consumable consumption (e.g. energy, water, nutrients, etc) and capable to operate autonomously for extended period of time.

The Reuse and Recycle technology area is a combination of technologies as well as a system engineering approach to design human exploration systems and components with a multi-purpose use goal; however, new technology is required to enable multi-purpose system/component designs. These technologies offer significant mass savings to the human exploration architecture by maximizing the use of every unit mass launched from Earth. Technology needs in this area includes (a) propellant and pressurant scavenging, (b) waste and trash management techniques to produce other products, and (c) repurposing of spacecraft system, components, and tools. Significant consumable mass is launched as propellants in the form or manager's reserve or safety margin. 


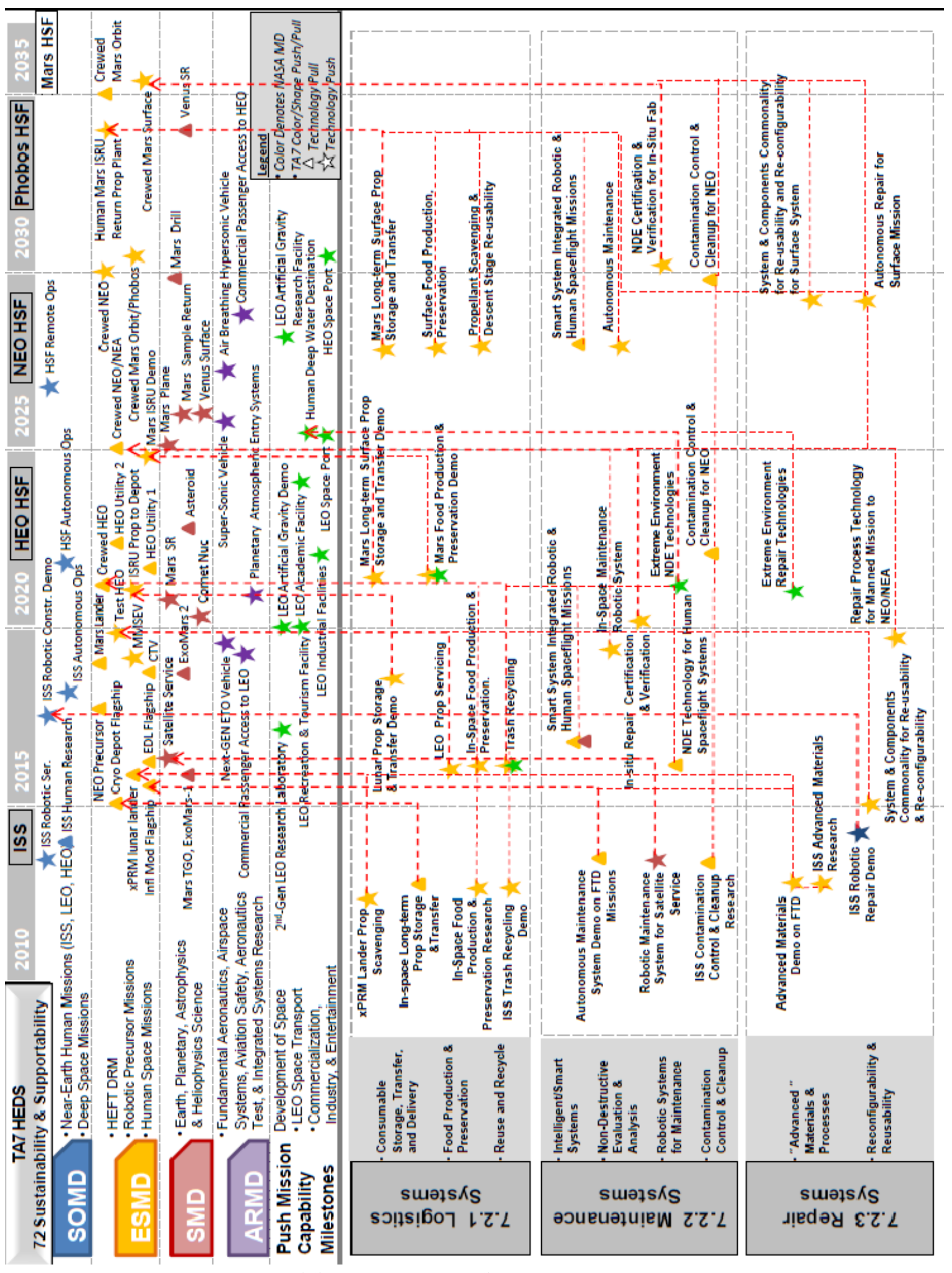

Figure 9: HEDS Sustainability and Supportability Level 2 Roadmap 

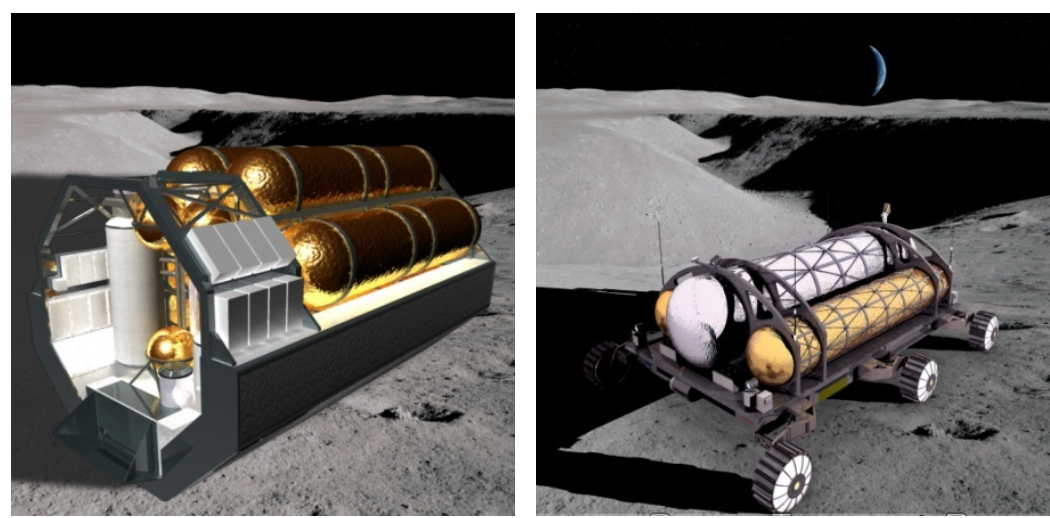

Figure 10: Stationary and Mobile Consumable Logistic Depot

Depending on the propulsion system, propellant scavenging can offer hydrogen, oxygen, and methane from main propulsion system and nitrogen, hydrogen, and oxygen from hypergolic reaction control systems. Also reclamation of helium pressurant would provide pressurant for hoppers or enable the reusability of descent stage (see 7.3 Advance Human Mobility Systems). Useful products such as fuel (e.g. methane) from human biological and nonbiological waste, radiation shielding from non-biological waste (currently used food wraps are mainly polyethylene) can be produced from waste, while managing waste during transit and at the destination. Managing waste during long-term transit and at the exploration destination can become a major task because of the health hazards associated with storing waste in a closed-loop environment and inefficient use of habitable volume inside the spacecraft, as well as planetary protection concerns. The reuse of spacecraft systems and components can result in significant mass reduction to the overall human exploration architecture. One specific example of repurposing is the re-use of pressurized logistic container for short term human occupancy, such as suit repair area, science labs, that otherwise would have taken space in "prime" habitable volumes.

\section{Maintenance Systems (HEDS TABS 7.2.2)}

A well-established maintenance program, as well as repair program discussed later, addresses sustainability and affordability aspect of the human space exploration program. The Maintenance Systems technology area includes those technologies needed to perform routine system evaluation, preventive maintenance, and corrective actions to human exploration systems. These technologies are (1) Intelligent/Smart Systems, (2) Non-Destructive Evaluation (NDE) and Analysis, (3) Robotic Systems for Maintenance, and (4) Contamination Control and Clean up. Nondestructive Evaluation and Analysis enables both intrusive and non-intrusive evaluation before, during, and after a maintenance task. Robotic Systems for Maintenance includes the technologies required for autonomous and semiautonomous robotic maintenance, as those shown in figure 11. Contamination Control and Cleanup includes those technologies needed to maintain systems from destination-specific environmental and human activity related contaminants.

The Intelligent/Smart Systems includes the technologies required to enable a system that selfmonitors, self-tests, and self-reconfigures. Technologies required for Intelligent/Smart System are: (a) software for integrated system health management (ISHM), (b) sensors for ISHM, (c) smart connectors, and (d) diagnostics networks. Intelligent/Smart Systems is a cross-cutting capability that requires a combination of software and hardware (e.g. sensors, data processors, etc) technologies. Specific Intelligent/Smart Systems for the HEDS TA is the need for a diagnostic network to enable an integrated management of multiple destination-specific systems, such as habitats, crew vehicles and mobility systems, power systems, ISRU systems, etc.

Non-destructive Evaluation and Analysis enables both intrusive and non-intrusive evaluation before, during, and after a maintenance task, and repair tasks as

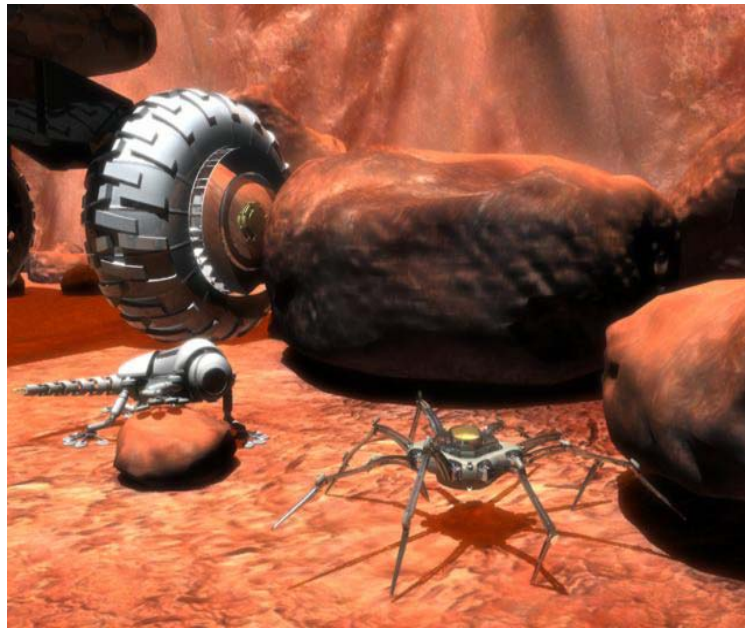

Figure 11: Maintenance and Repair Microbots 
applicable. NDE and Analysis technologies specific to human space exploration systems include: (a) pressurized vessel structure integrity, (b) pressurized system leak detection, (c) human mobility system diagnostics and checkout, and (d) maintenance tools, (e) maintenance and repair tasks, and (f) in-situ manufactured parts/components verification and certification. The pressurized vessel structure integrity includes the evaluation and analysis of structural and skin components (including windows) of habitable volumes and pressurized vessels such as tanks, fluid lines, seals, and others. Pressurized systems leak detection include macro and microscopy leaks as they apply to spacesuits, fluid handling equipment (chemical processes, propulsion systems, pressurized consumable depots), and pressurized habitable volumes. For advanced human mobility systems (see 7.3), NDE and Analysis techniques for EVA, surface and off-surface mobility systems are required to determine the integrity of the mobility system at the component and system level, as discussed in Section 7.3.2. Maintenance tools, maintenance and repair tasks, and in-situ manufactured parts must follow the same verification and certification processes used during ground processing of manned-rated systems. Space-rated version (i.e. minimum power, volume, mass, process time, etc) of those specialized process and techniques, such as tool, maintenance and repair tasks test, and part manufacturing quality control, must be developed in order to improve crew safety and mission success.

Robotic Systems for Maintenance includes the technologies required for autonomous and semi-autonomous robotic maintenance. These technologies include precision manipulators, high level of dexterity manipulators and systems, and specialized maintenance robotic tools. Autonomous robotic maintenance systems are capable of increasing the efficiency of the mission by minimizing the time crew members spend performing maintenance tasks, which can be expected to be significant time during long-duration deep-space human exploration. Technologies in this area should have a long-term goal of developing artificial intelligent robotic systems capable of performing maintenance tasks autonomously. A good example of such technology would be an army of specialized microbots, each performing specific tasks, that when combined can perform a wide variety of maintenance tasks.

Contamination Control and Cleanup includes those technologies needed to maintain systems from destinationspecific environmental and human activity related contaminants. Specific technologies in this area are contamination detection and maintenance (corrective action) of hardware (e.g., spacesuits) to protect habitable volumes.

\section{Repair Systems (HEDS TABS 7.2.3)}

Deep-space human exploration mission will require the capability to repair systems and components that may fail at anytime during the duration of the mission to increase crew safety and mission success. Repair System technology area includes technologies that address "wear-and-tear" repairs as well as technologies required to perform minimum or non-intrusive repair. These technologies are categorized into (1) Advanced Material and Processes, and (2) Systems Reconfigurability and Reusability. Autonomous robotic systems for repair are a required technology under Repair Systems; however, it is understood that similar, if not the same, robotic technologies (e.g. manipulators, tools, etc) discussed under Maintenance Systems section are applicable to Repair System with a repair implement. The Advanced Material and Process technology area includes technologies required to perform passive repairs (no human or external source intervention; self-healing) and active repair (requires some human or external source intervention; welding). System Reconfigurability and Reusability requires technologies (software and hardware) to reconfigure integrated system to isolate the area that requires repair without affecting the availability and operation of the entire systems.

The ability to repair requires the development of repairable materials and processes to perform such repairs. The Advanced Material and Process technology area includes technologies required to perform passive repairs and active repair. Passive repairs are defined as those that do not require any intervention to perform the repair, such as the case for self-healing technologies for small flexible application (e.g. wire insulation, textile, etc) and larger structural application. Active repairs are those requiring some action to perform the repair. Technologies to perform active repairs include welding technologies, surface repairs, and rapid prototyping technologies. Self-monitoring advanced materials with integrated nanosensors to detect stress, fracture, or crack for early repair indication are required to prevent failure of the material.

The ability to repair complex systems requires that systems be designed to allow for minimum intrusive repairs as well as for reusability of critical components. System Reconfigurability and Reusability requires technologies (software and hardware) to reconfigure integrated system to isolate the area that requires repair without affecting the availability and operation of the entire systems. Reusability and redundancy of mission critical hardware is a needed technology to allow for non-critical components and systems to be use to repair or to replace failed mission critical components or systems. A good example of this technology would be the use of an avionics component from a spent propulsion system to repair a critical avionic component on a habitat. Although the ability to scavenge components is a design feature (highly dependable of the location of the component), technology development is needed to have 
interchangeable, in-situ reprogrammable/reconfigurable components and systems to allow for reconfiguration and reusability.

\section{Advanced Human Mobility Systems (HEDS TABS 7.3)}

Astronauts traveling to new destinations will experience an environment fundamentally different from that in LEO. Extended trip times will increase the demand for protective shielding from radiation, potential bombardment by micrometeoroids, and extreme thermal environments. To accomplish NASA's mission objectives and reap greater science reward mobility systems will be required to extend our reach outside of the atmospherically controlled habitable accommodations of spacecrafts. Spacesuits with provisions for high-pressure oxygen supply are required for breathing and maintaining safe pressure and thermal environment around the body. To explore and work in space, mobility aids will be needed to get to areas on and off-surface that might otherwise not be accessible. This includes EVA tools and translation aides that improve the safety and effectiveness of crews, utilizing land vehicles, propulsive and non-propulsive systems for aerial travel over varying distances, and construction of infrastructure to facilitate transporting both crew and cargo. Globally, lightweight, high-strength, materials capable of tolerating temperature extremes are applicable, as they will improve performance and reduce launch mass. Technology milestones are mapped to provide an off ramp approach for developing capabilities that support human research on orbit in LEO or crewed missions to NEOs and Mars. In most cases current efforts can be leveraged to accelerate technology development commensurate with available funding and the development of capabilities to launch assets.

The HEDS mobility roadmap further establishes a sustained presence in space, i.e., going beyond LEO. The timely maturation of technology investments outlined for mission insertion will build on current capabilities and establish performance improvements that will allow astronauts to achieve mission objectives (fig. 12). The Advanced Human Mobility Systems Level 2 roadmap shows the detailed fundamental and applied technologies mapped to the pull and push Mission Directorate missions (Fig. 13).

\section{EVA Mobility (HEDS TABS 7.3.1)}

Significant progress can be gained from observation of past EVA operations, noting inefficiencies that can be reduced through successful pursuit of targeted technologies. One such technology is a lightweight, more adaptive spacesuit, characteristically more ergonomic in design to facilitate ease of entry and egress. Spacesuits incorporate a number of subsystems, including advanced pressure garment suit materials, life support, power, and communications technologies. Prior to conducting EVA at reduced pressure ( $\sim 4.3$ psia), extensive pre-breathe of $>95 \%$ oxygen concentration is needed to remove nitrogen that could otherwise be released from body fluids as painful gas bubbles when pressure is reduced. Incorporating pressure management improvements into the suit Portable Life Support System (PLSS) and data systems are critical technologies that will decrease preparation time and maximize time for operations.

Requirements for mitigating dust or other contaminants while performing EVA, and for enabling quick access to a safe haven for radiation protection or in the event of a contingency must be considered for HEDS missions. Traditional airlocks either waste a significant amount of consumables with each EVA or require excessive time to reclaim the gas. To facilitate ease of operations and mitigate risks to the habitat while performing EVA for HEDS missions, an alternative airlock solution is needed. Current airlock designs waste atmosphere and are not compatible with dust, biological, or chemical isolation. Dust contamination will be a significant issue on the surface of the Moon, Mars, and potentially on NEO missions. A primary challenge is to decrease the time associated with vehicle or habitat egress and ingress by fifty to seventy-five percent, reducing the gas loss during depressurization, and decreasing the potential of contaminating the cabin due to bringing in dust or $\mathrm{CO}_{2}$. This objective could be achieved with a suitport, suitlock, or some type of advanced airlock. In a suitlock system, an environmentally protective spacesuit with a sealing surface coincident with the habitat is accessible from inside of the habitat to facilitate entry and egress. It is sealed upon entry into the spacesuit and separable from the vehicle for EVA via mechanical interface and latches. A suitlock or similar logistical approach to EVA would lessen requirements for depressurizing the habitat and sending the full crew on EVA. The development of inflatable seals, dust-proof electrical, oxygen and water connectors between vehicle(s) and the suit will be needed for missions to dirty environments, which includes the Moon, Mars, or asteroids. Suitport, hybrid suitport/suitlock, and airlocks will be kept in the trade space until DRMs are explicit. Pressurized demonstrations of a suitport are needed beginning in 2011 to support a flight demonstration prior to 2018 . 


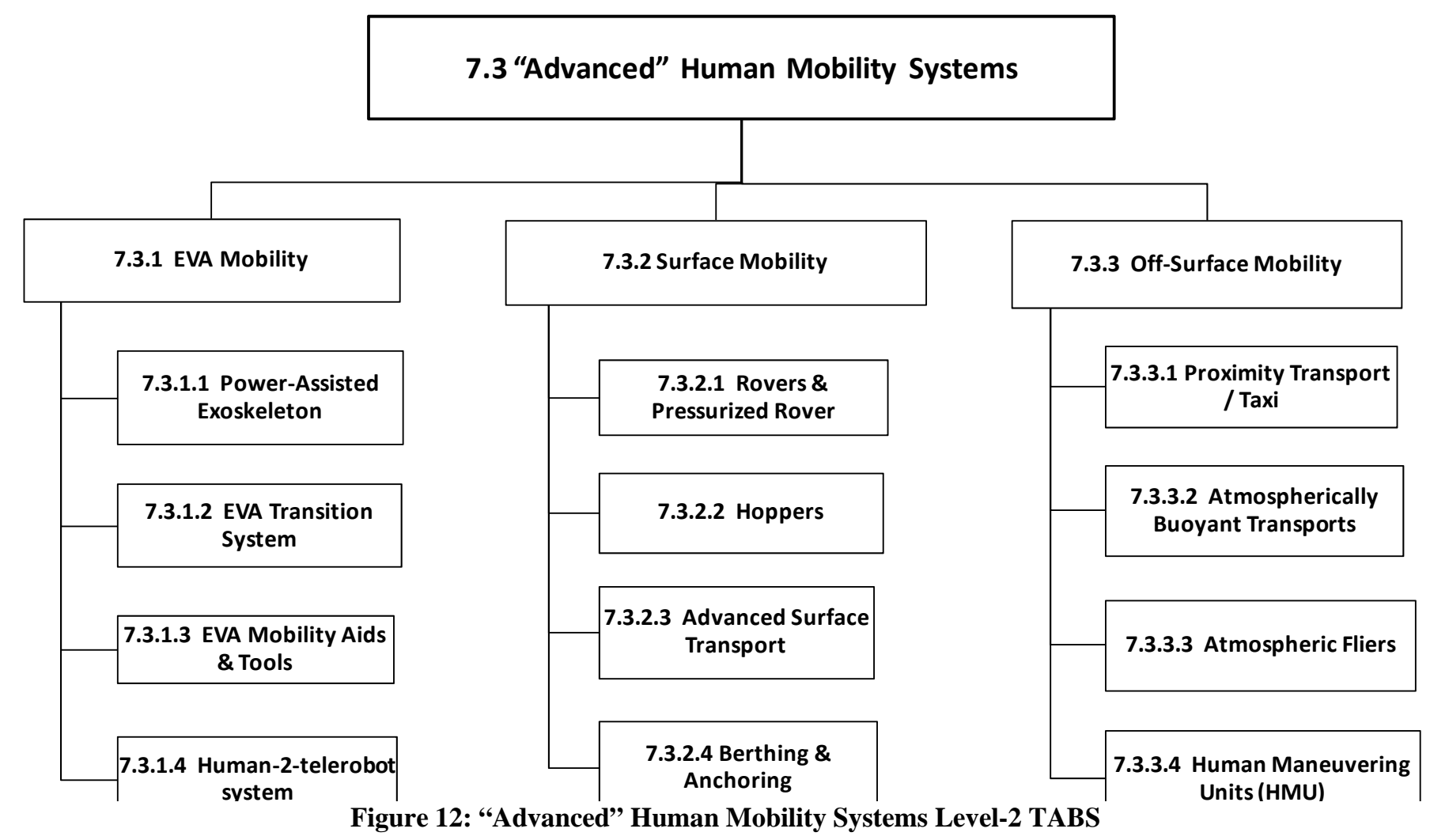

Further systematic block upgrades in materials, applying biomechanic improvements for ease of movement and more optimized interfaces to airlocks or suitlock design could evolve suit technology into a more adaptive solution, enabling humans to walk and work on the surface Mars and other destinations. Much of the technology required for the evolutionary development of space suits is within the scope of TA6 - Human Health, Life Support, and Habitation Systems. Humans, and more specific to this description, astronaut crews, are limited in the amount of weight that they can pick up and carry. Mobility aids and tools will be needed to provide capabilities such as lighting, data transfer, sensory feedback, safety gear, and task specific devices that are needed to achieve mission objectives and expedite movement about remote locations. Fielding astronauts outfitted with a wearable robotic system, or exoskeleton, would augment their natural abilities through actuated power to aid its human wearer in performing normal tasks. Applying human-to-telerobotic interfaces and power-assisted robotic systems design could enable automated operations and exoskeleton capabilities with increased strength that will minimize the number of crew required and effectively reduce cost to achieve mission objectives. Current research in other government agencies is aimed at military applications like increased strength and traversing unpredictable terrain. Development of sensory feedback to monitor performance coupled with recent advancements in lighter, stronger carbon fiber composites or alternative materials may further enable exploration capabilities.

\section{Surface Mobility (HEDS TABS 7.3.2)}

For a number of NASA's mission architectures humans must be able to work on the surface of terrestrial destinations. To navigate the rough surface terrain improvements in rover component and subsystems will be needed. Component development of wheels, drive trains and similar high duty subsystems are needed to meet the longer life requirements (approx. 10,000 km) for pressurized and non-pressurized rover systems that may carry up to one hundred times their own weight. Advancements in non-conventional models used to estimate drawbar pull and traction in loose planetary soils with uneven or uncertain terrains is needed to better predict performance and influence rover design. Operating in harsh environments, i.e., vibration, peak torques, regolith/dust, and $40 \mathrm{~K}$ to $400 \mathrm{~K}$ temperatures, compounds the difficulty. Evolving to wheel-on-limb designs will provide multifunctional capabilities that can automate operation and minimize crew hours. Advanced rovers will likely have alternative power sources, some of which will be rechargeable and would benefit from automated docking and berthing mechanisms that facilitate ease of charging batteries. Interdependencies between TA4 - Robotics, Tele-Robotics, 


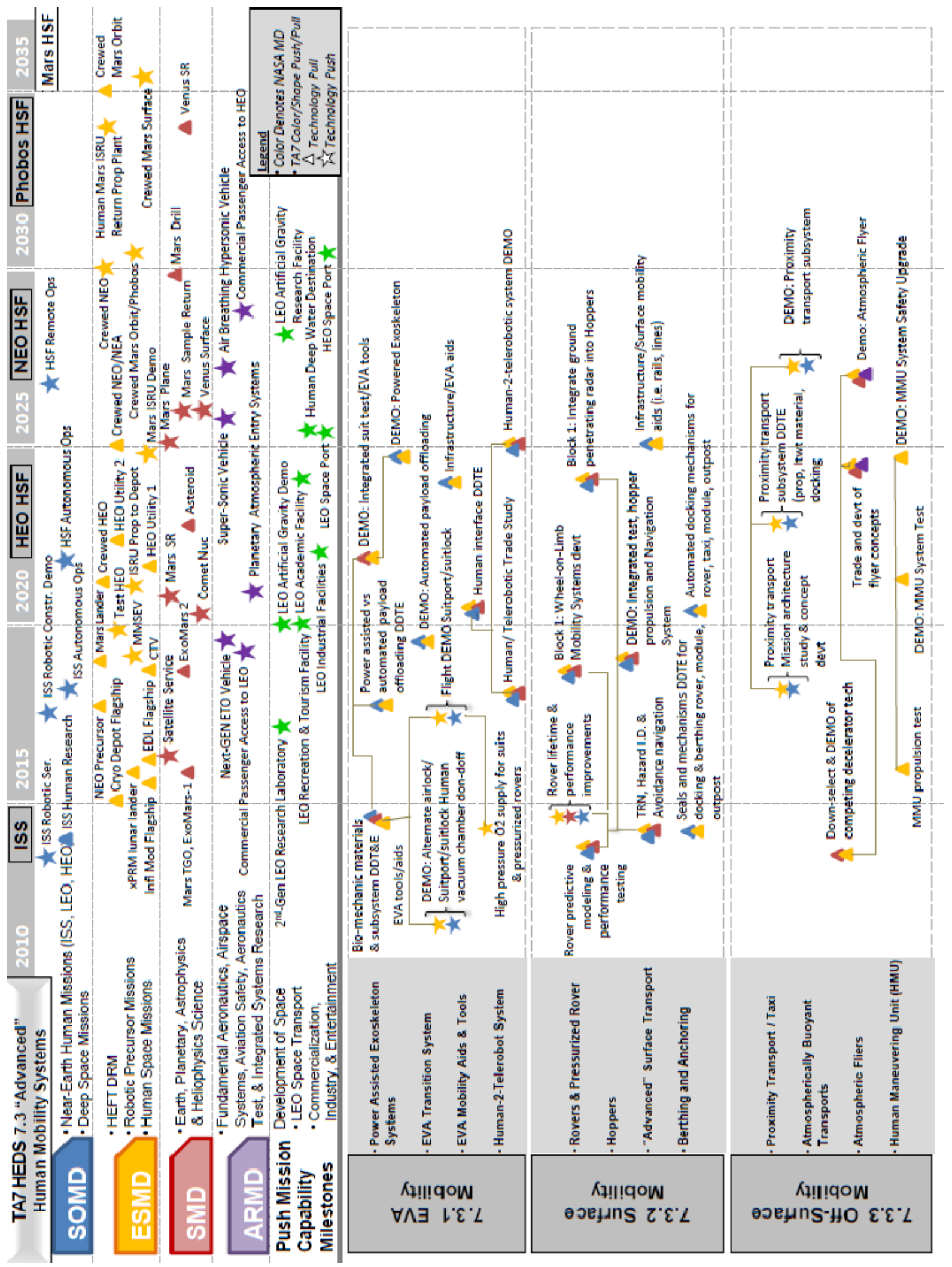

Figure 13: HEDS “Advanced” Human Mobility Systems Level-2 Roadmap 
and Autonomous Systems and TA7 are necessary to advance technologies forward, incorporating algorithms, avionics, and automation mobility systems such as rovers.

To navigate and access extreme land features such as craters 'hoppers' or fueled planetary explorers that hop can transport crew \& cargo over short to moderate distances on other bodies (including NEA, NEO, Moon and Mars). Propulsion and hazard identification and avoidance using LIDAR (Light Detection and Ranging) are well-suited technology developments that will provide capability for terrain relative navigation (TRN). Surface exploration will require a new complement of tools for sample acquisition and in-situ analysis. New EVA mobility systems are also needed to perform microgravity surface missions on NEOs. EVA mobility items include tools and equipment that attach to a spacesuit, such as lighting and cameras, sensors, task-specific devices and safety gear. This includes EVA translation aids required to enable an EVA crewmember to safely translate, react to forces and loads, and restrain them in order to do useful work in reduced and microgravity environments. Once established at the destination, infrastructure in the form of locally constructed rails and zip lines or other conveyance aids that enable movement of larger assets will assist the crew in traversing the surface. More definitive infrastructure would be established through HEDS construction once at the destination.

\section{Off-Surface Mobility (HEDS TABS 7.3.3)}

The need to transport crew or cargo around a destination is not limited to the surface operation. Atmospherically buoyant transports such as balloons or other forms of deployable airships have been identified for missions to destinations with an atmosphere. They offer reduced mass and volume to improve payload mass fraction. Atmospheric fliers such as gliders and conceptual 'wing-suits' provide still more options for crew to travel to and from surface locales. Possibly a more immediate option, the development of human maneuvering units (HMUs) or 'jetpack' offers a means for EVA crewmembers to move about in areas that other alternatives do not enable. To date personal flight devices have been tested on prior NASA space missions with varying results for the 'worst case' scenario of an EVA tether failure. Provided they can be easily refueled and stowed, further development of jetpacks would improve safety and may provide capability to translate between orbiting space vehicles as an efficient solution for individuals to perform maintenance, assembly, and repairs. On a larger scale taxis offer the ability to ferry crew and cargo over longer distances to a number of docking/berthing options with other vehicles or modules on and off surface. Techniques to anchor the vehicle to the destination are addressed under TA9 - Entry, Descent, and Landing Systems. Mechanisms and techniques for anchoring personnel or docking and berthing modules or vehicles can leverage ISS experience but require further development and demonstration for ESMD and ARMD HEDS locations.

\section{D. “Advanced” Habitat Systems (HEDS TABS 7.4)}

One area of HEDS is the Advanced Habitat Systems (AHS) technology area WBS 7.4. AHS covers the deep space habitat for (beyond cis-lunar) transportation and sustained presence of humans to and at various destinations. The AHS relies on many of the other technology area's fundamental research and technology development in order to develop into "applies" technologies applicable to destination capabilities. The purpose of AHS is to enable the capability of human Habitat systems at destinations far from Earth, thereby increasing crew productivity, safety, and successfully conduct mission objectives. The AHS WBS is further broken down into two areas as follows: 7.4.1 Integrated Habitat Systems and 7.4.2 Habitat Evolution (figure 15). Each of these areas will be discussed in more detail below. The AHS Level 2 roadmap shows the detailed applied technologies mapped to the pull and push Mission Directorate missions (Fig. 16). As previously mentioned the human mission technologies milestones are representative of TRL-6 at PDR 6 years prior to the mission launch. Therefore the mission and technology milestones are off-set accordingly.

Advanced habitats for exploration destinations include deep-water ocean destinations in support of the evolutionary approach to deep-space destination such as HEO, NEA, NEO, interplanetary travel, and planetary missions. AHS technologies address both evolutionary and revolutionary game-changing technologies that will not only fulfill "pull" missions, but also "push" missions. The AHS technologies focus on enabling destination capabilities in an incremental "Block" five-year (5 yr) cycle of maturation. Each "block" builds on the previous technologies and adds additional capabilities. Block-1 AHS technologies focuses on capabilities that enhance the Inflatable Module Flagship demonstration planned for ISS. Block-2 AHS technologies focuses on capabilities that enhance HEO crewed missions. Block-3 AHS technologies focus on capabilities that enhance NEA and NEO crewed missions. Block-4 AHS technologies focus on capabilities that enable human missions to Mars vicinity. This 
includes enabling a crewed mission to Phobos, pre-deployed surface habitat on a cargo mission, and the interplanetary crewed mission to Mars Orbit (figure 14).

\section{Integrated Habitat Systems (HEDS TABS 7.4.1)}

This Level 3 technology area includes integrated habitation systems capabilities to enable long-duration and deep-space human missions that increase crew productivity, increases crew and mission safety, and reduce mass, power, and volume needs. This area includes technologies in four areas of Advanced Fabric Materials, Internal Systems \& Outfitting, Light Weight Windows, and "SMART" Habitats. Most of these technologies identified within the portfolio are TRL $\sim 3-4$.

For long-duration deep-space missions the crew will require new materials and colors that can be used in lowpressure high oxygen environments while being non-toxic. This will support the crew's well being both physiologically and psychologically. The same is true for the internal systems, advanced windows, and "smart" habitats. Whereas the missions are still being defined this strategic approach allows a flexible path of technology development.

Advanced fabric materials include new low-toxicity fireretardant textile materials have been identified being required for operation in a low pressure ( $\sim 8.3$ psia, 841 kilopascal) and high oxygen $(\sim 30-32 \%)$ internal environment to enable crew psychological well-being and safe operations of human interplanetary spacecraft and surface habitats on longduration missions identified by long-duration deep-space human missions. This area includes technologies for advanced textiles that can be used internal to the spacecraft in low PSI

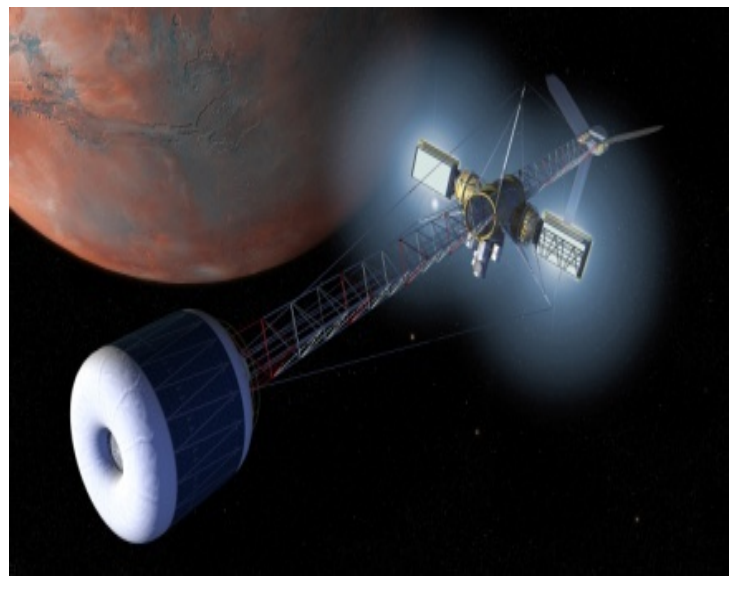

Figure 14: Deep Space Mission and high $\mathrm{O} 2$ environment that will allow multiple color choices, low toxicity off-gassing, and is flame retardant for long-duration deep-space human missions. Examples of technologies required include low toxicity off-gassing; flame retardant; multi-color; self cleaning; antimicrobial surfaces; integrated sensors; bio-technology coating for toxicity detection; bio-sensing; bio-technology coating for illumination; bio-technology coating for self cleaning; integrated radiation protection; automated color / image changeability; and integrated power management and distribution for low power wireless distribution.

Internal Systems \& Outfitting: Internal Systems \& Outfitting have been identified being required to enable implementation of mission milestones identified by long-duration deep-space human missions. This area includes internal systems \& outfitting technologies to enable long-duration and deep-space human missions that increase crew productivity, increases mission safety, and reduces mass, power, and volume needs. Examples of technologies required include Deployable Outfitting and Internal Structures; Sound Absorption; Connectorless Utilities Integration (Power, ECLSS, Data); Safety Caution, Hazard and Warning systems; Lighting Natural (Fiber Optic); Crew quarters, Galley, Relaxation \& Entertainment. For acoustics, the primary challenge is developing low-noise (40-50 dBA) fans and pumps to move the air and heat-exchanging fluids in spacecraft ECLSS.

A substantial amount of research, modeling techniques and tools related to aircraft turbofan noise reduction exists, but these tools have not been applied to or validated at the significantly reduced sizes, flow-rates and pressure rise requirements (i.e. Reynolds numbers) needed for spacecraft ECLSs. Noise cancellation technology may also be employed as a mitigation strategy in the event that equipment failures result in noise levels that are hazardous or that interfere with communication or sleep.

Light weight durable windows have been identified being required to enable implementation of mission milestones identified by human spaceflight. This area includes technologies for light weight durable windows to be used on long-duration deep-space human missions. Applications include rovers, habitats, and laboratories. Examples of technologies required include multi-purpose coatings; nano-technology; light-weight high-strength translucent materials; self-repairing, self-healing; bio-technology coatings; polycarbonates; transparent aluminum; induced variable translucency ('smart' glass); and seals.

Intelligent "SMART" Habs have been identified as enabling sustained human presence of long-duration deepspace human missions by increased autonomy and operations. This area includes technologies of intelligent "SMART" structures such as integrated software controls, sensors, self-repairing, bio-technology, and nanotechnology for unpressurized and pressurized structures to sustained human presence of long-duration deep-space 
human missions. Applications include habitats, laboratories, unpressurized shelters, under-ground facilities, storage container or shelter, telescopes, rovers, antennas, and hybrid suitlocks.

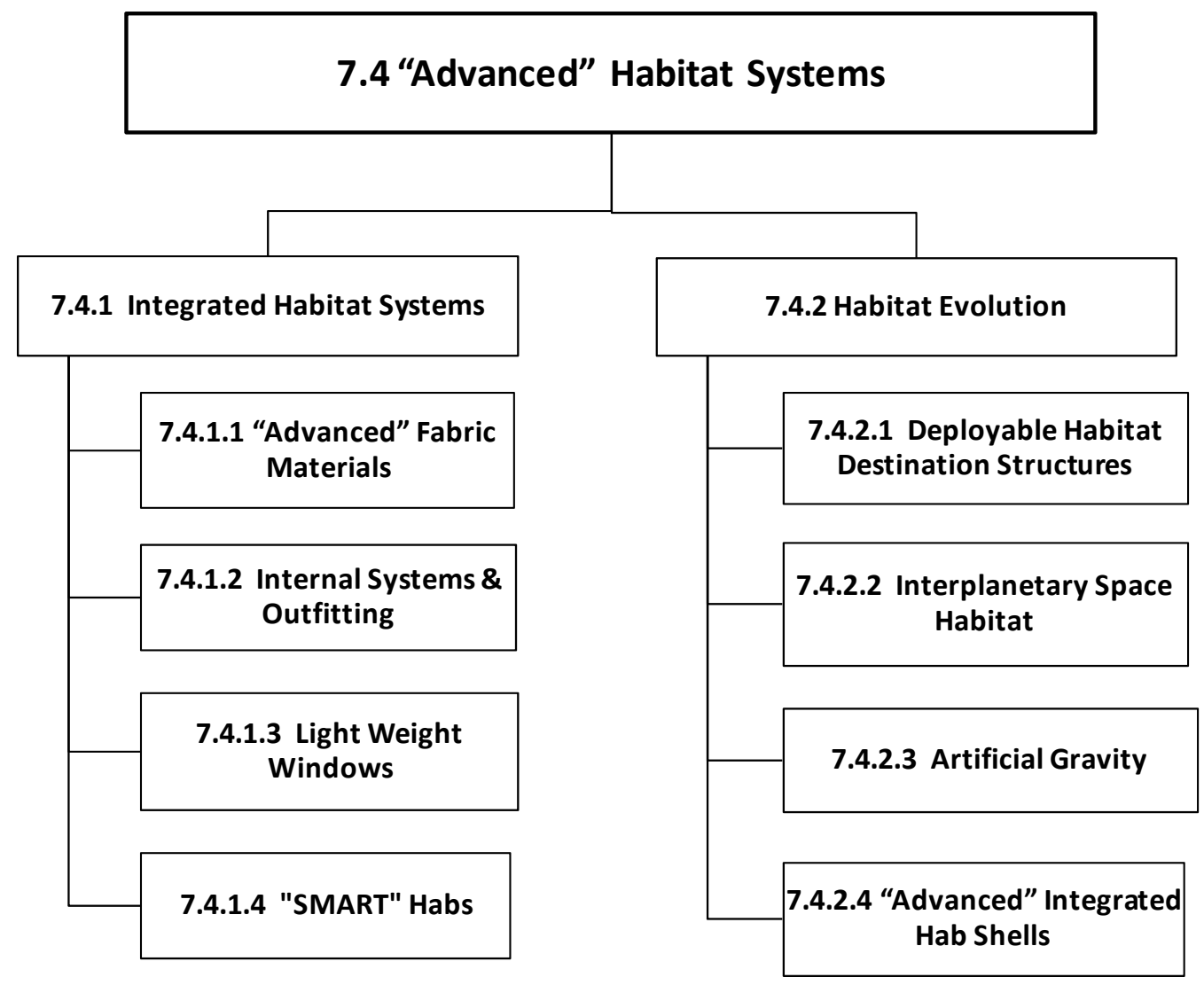

Figure 15: TA7 HEDS “Advanced” Habitat Systems Level-2 TABS

\section{Habitat Evolution (HEDS TABS 7.4.2)}

This area includes advanced habitat technologies that will enable deep-space habitats capabilities in support of a sustained human presence in space. Habitat Evolution includes human-occupied structures capability to enable long-duration and deep-space human missions that increase crew productivity, increases mission safety, and reduces mass, power, and volume needs. This area includes technologies in four areas of Deployable Habitat Destination Structures, Interplanetary Space Habitat, Artificial Gravity, and Advanced Integrated Habitat Shells.

Deployable Hab destination structures include light-weight inflatable and rigidized deployable structures that have been identified being required to enable implementation of mission milestones identified by human spaceflight. This area includes technologies of "expandables" for unpressurized and pressurized inflatable and rigidized structures to enable long-duration deep-space human missions. Applications include habitats, laboratories, rovers, unpressurized shelters, large telescopes, antennas, and hybrid suitlocks (Fig. 17). Examples of technologies required include Long Life Durable Textile Materials; Air-Inflated, Air-Supported, Self-Rigidized air beams; Seals; Adhesives; Joints and Seams; high-Efficient packaging; Secondary Structure attachment to primary inflatable; SelfRigidizing Structures; Patching \& Repairing Inflatables; Self Healing; integrated sensors into tensile fabric for Maintainability and Serviceability; Connectorless inter-module utility transfer; Nano-Tech Integration; and BioTechnology Integration.

Interplanetary spacecraft transport vehicles have been identified being required to enable implementation of mission milestones identified by long-duration deep-space human missions. This area includes Interplanetary Space Habitat technologies that enable long-duration deep-space human transportation. Examples of technologies required include radiation protection; autonomous operation; robust \& reliable components; inflatable structures; selfrepairing; Integrated system management; low power lighting (LED, solar optic); and advanced textile fabrics. 


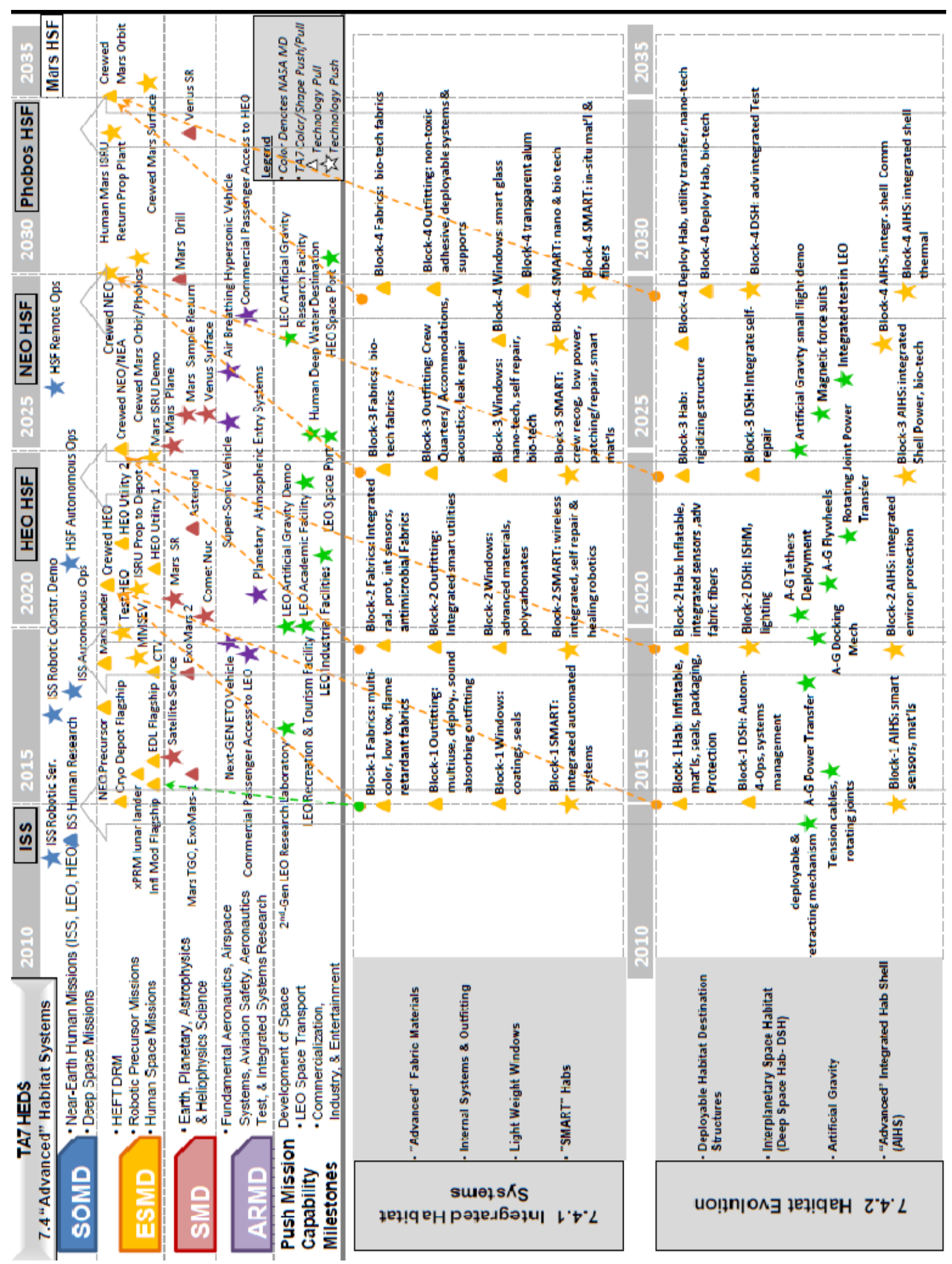

Figure 16: HEDS “Advanced” Habitat Systems Level-2 Roadmap 
Artificial Gravity spacecraft has been researched for years as an option for reducing the detrimental effects of long-duration 0 -g on the human physiology. It would also benefit LEO and HEO commercial facilities. Artificial Gravity includes technologies and research of Artificial Gravity spacecraft for long-duration deep space missions. Examples of technologies required include Coriolis counter-effects technologies; deployable and retracting mechanism for A-G spin-up \& down in zero-G; power and/or propulsion for spin-up/spin-down; tethers (momentum exchange); light weight high-strength tension cables; slip-rings for high efficient power transfer on rotating joints; counter rotating docking mechanism; and magnetic force suits (body suits that would experience a downward force due to localized magnetic field).

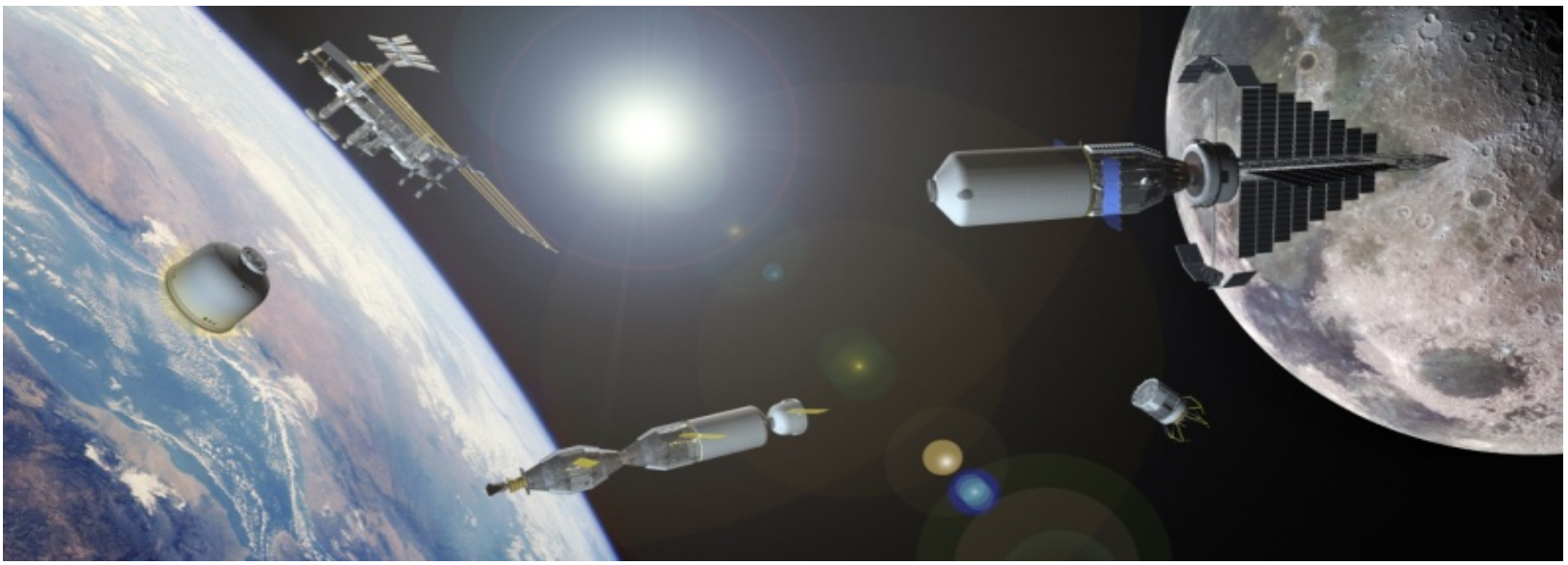

Figure 17: Commercialization of Low Earth Orbit Space

Advanced Integrated Hab Shells have been identified as enabling sustained human presence of long-duration deep-space human missions by increased shell efficiency, increased protection, crew safety, and reduced over-all mass. This area includes technologies of Advanced Integrated Habitat Shells such as integrated protection, integrated power, thermal, and communications technologies for pressurized human occupied shells to enable sustained human presence of long-duration deep-space human missions. Examples of technologies required include Integrated Environmental Protection w/ Shell; Integrated Power Systems w/ Shell; Integrated Thermal Systems w/ Shell; Integrated Communications Antenna Systems w/ Shell; nano technologies; integrated sensors; advanced materials (polycarbonates, hybrid alloys, rigidization foams); and bio technologies.

\section{E. Mission Operations \& Safety (HEDS TABS 7.5)}

The main task of mission operations is to manage space missions, usually from the point of launch through the end of the mission; the 'start-to-finish' development and delivery of highly complex of robotic and human spaceflight operations. To that end, mission operations entities across NASA centers provide time-appropriate failure analysis and response to protect crew and spacecraft safety in order to achieve mission objectives.

Currently, NASA is beginning to consider human missions to near-Earth objects or NEOs (by strict definition, an NEO is a solar system body that includes both comets and asteroids with a perihelion of $1.3 \mathrm{AU}$; for purposes throughout this document, astronauts would only likely visit bodies that are asteroidal in nature). The idea of sending human explorers to asteroids is not new. Piloted missions to these primitive bodies were first discussed in the 1960s and paired upgraded Saturn V rockets with enhanced Apollo spacecraft to explore what were then called 'Earth-approaching asteroids.' Since then, several other studies have examined the requirements for sending humans to such primitive bodies in close proximity to Earth.

The drivers for piloted missions to NEOs are political, scientific, and programmatic. Such missions would reinforce U.S. human spaceflight leadership while greatly increasing humanity's knowledge and experience in such areas as: solar system evolution and formation; origins of life on Earth; deflection strategies for potentially hazardous NEOs; development of safe, reliable long-duration deep space exploration and operations; designs for more capable exploration architectures; and establishment of in-situ resource utilization (ISRU) efforts. Visiting the Moon and NEOs are a feed forward to the vicinity of Mars and beyond. The Mission Operations and Safety TABS (Fig. 18) and Level 2 roadmap shows the detailed fundamental and applied technologies mapped to the pull and push Mission Directorate missions (Fig. 19). 


\section{Training (HEDS TABS 7.5.1)}

Inside or outside the spacecraft, the most valuable asset of any deep space expedition is the astronaut crew. The crew conducts a full suite of engineering and science activities, while adapting to unexpected situations with insight and flexibility. The key to their success and that of the mission is 1) training and 2) on-board autonomous systems. Robust autonomous systems are a key consideration for voyages well beyond the Moon when the time-to-criticality (TTC) of any situation, not just a system fault, is less than the time needed to communicate with mission control. For the Moon, the one-way light time (OWLT) is just under 1.5 seconds; for missions to LaGrange points (i.e., SEL1 or SEL2) OWLT is $\sim 5$ seconds; for missions to the likely NEOs human may explore someday OWLT is $\sim 20$ to 30 seconds. The NEO mission concept begins to introduce a tipping point in which the crew must be much more self-reliant, especially if a fault results in loss of communications - then, the mission and crew are in a situation in which mission control cannot even be notified of the problem [via voice or even via telemetry]. In this case, the crew must execute whatever recovery procedures they have in place to restore communications and then work to resolve the main problem. This is where recurrent on board virtual training is essential. Psychologically, it will keep the crew purposely occupied and engaged in the mission and could very well save their lives.

On-board virtual reality training could also provide refresher training for the practicality of tasks to be performed at the NEO (or Phobos or even the Martian surface) when it comes to planetary surface operations. The EVA crew's EVA capabilities make possible a broad array of surface operations and sample collection tasks. An astronaut's ability to anchor, traverse, and collect macroscopic samples in geological context from several NEO terrains would rapidly produce a wealth of data on regolith density and particle sizes, potential space weathering effects, impact history, and mechanical properties. The training before and the subsequent adaptations to the final servicing mission to the Hubble Space Telescope (STS-125) is a prime example of the flexibility and adaptability of human crews performing complex tasks under difficult conditions.

As humans venture further from Earth and the one-way light time (OWLT) increases, along with potential communication disruptions, the crew must become a much more self-reliant and systems savvy 'mini-mission control.' Given the anticipated upgrades in ISHM software, spacecraft avionics and hardware, the paradigm for crew training must evolve and dramatically change from the methods currently utilized for shuttle and ISS. The crew will need to train to autonomously handle a wide range of nominal and off-nominal situations for durations of tens of seconds to minutes without MOD support. They will need to train to rely more on on-board intelligent software for situational awareness rather than constant real-time tactical MOD support. Given the long duration of missions, ongoing training will need to be an integrated part of the regular schedule during cruise phases of a mission - without real-time support from ground-based trainers.

This implies that training capability will need to be a built-in function of the onboard avionics and software, including simulated fault injection and detailed simulation of later mission phases such as proximity operations near a NEO. Crew training, both before flight and during flight, will benefit from advances in virtual reality; as well as being informed by other governmental agencies' approaches to in-situ training. Human factors advances will need to provide guidance on both pre-flight and during-flight training for human-machine operations, including just-in-time training. Training and simulation software will need to be incorporated into the flight software architecture form the earliest stages of design, rather than be an afterthought for separate ground-based facilities.

\section{Environmental Protection (HEDS TABS 7.5.2)}

The greatest environmental risk to human spaceflight beyond the Earth's magnetosphere is radiation. Ionizing radiation, galactic cosmic radiation (GCR) and solar cosmic radiation (SCR), is a significant risk to astronauts for all long-duration missions beyond LEO regardless of destination (lunar surface, Mars or NEO). Many of these high energy elementary particles penetrate spacecraft, pressure vessels and space suits (generating shrapnel-like secondary radiation in the process) as well as organs, cells and DNA of human occupants inducing degenerative changes usually associated with accelerated aging (extracellular matrix remodeling, persistent inflammation, oxidative damage, cataracts and damage to the central nervous system). DNA damage results in increased mutation rates, genomic instability, cancer induction and activation of latent tumors.

Current spacecraft (as well as the forthcoming Crew Transfer Vehicle) rarely provide more than $10 \mathrm{gm} / \mathrm{cm}^{2}$ of shielding. For the sake of comparison, Apollo-era LEM had $<5 \mathrm{gm} / \mathrm{cm}^{2}$. Modern spacesuits provide $<1 \mathrm{gm} / \mathrm{cm}^{2}$. The space radiation environment is characterized by an undulating baseline of GCR interrupted by solar events of varying intensity and duration. Solar disturbances can unleash tremendous bursts of high-energy protons and heavy nuclei in coronal mass ejections (CME) delivering significant doses of radiation over the course of a few hours. Radiation exposure was not an operational problem for Apollo missions to the Moon. But, it is for long-duration deep space missions to the realm of NEOs and beyond. 
Regardless of how 'impractical' shielding might be, it is clear creative shielding approaches will be part of the solution. The relationship between differences in shielding effectiveness of various parts of the spacecraft and duties, movements and locations of individual crewmembers could partially explain observed variations. Preferential placement of equipment, food, water and waste material in long-duration spacecraft could facilitate radiation protection. Efficient radiation resistant materials such as polyethylene could play a role in lining sleep stations or internal 'shelters.' Outside-the-box strategies could include external shielding approaches such as storable propellant placement (perhaps during coast phases of flight) or even the use of NEO regolith 'sandbagging' to protect crew compartment(s) during proximity ops and return phases of the mission. Another promising research priority is the development of radio-protective pharmaceuticals that could be taken as a preventive or in response to a significant increase in radiation (such as an SPE).

Long-duration ECLSS without the possibility of resupply or timely abort to Earth is another challenge. This encompasses a wide range of environmental risks from meteor penetration to fire to toxic gases, as well as gradual ECLSS system degradation. Missions will need to carry enough extra consumables to deal with these contingencies, but any delay in detection or response to environmental problems will magnify both risk and the mass penalty of required extra consumables. Intelligent system health management including prognosis as well as on-board diagnosis are capabilities that will be needed. In addition, a lifeboat capability sufficient for months while a rescue mission is launched is a necessity. As was done serendipitously in Apollo 13, reconnaissance and other vehicles can double as a lifeboat in emergencies - or lightweight deployable structures. Enhancing capabilities could include human factors such as methods to slow human metabolism.

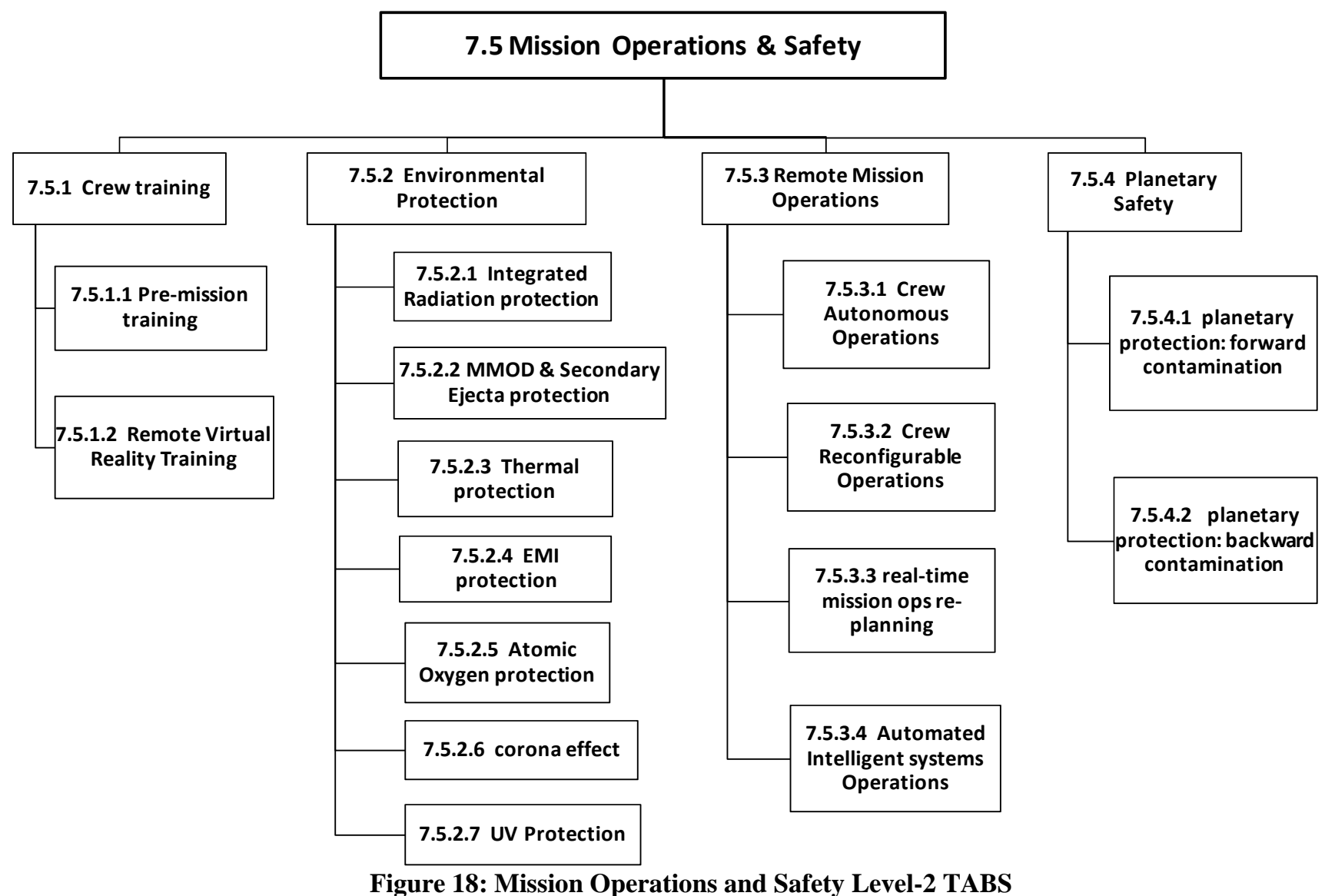

Figure 18: Mission Operations and Safety Level-2 TABS 


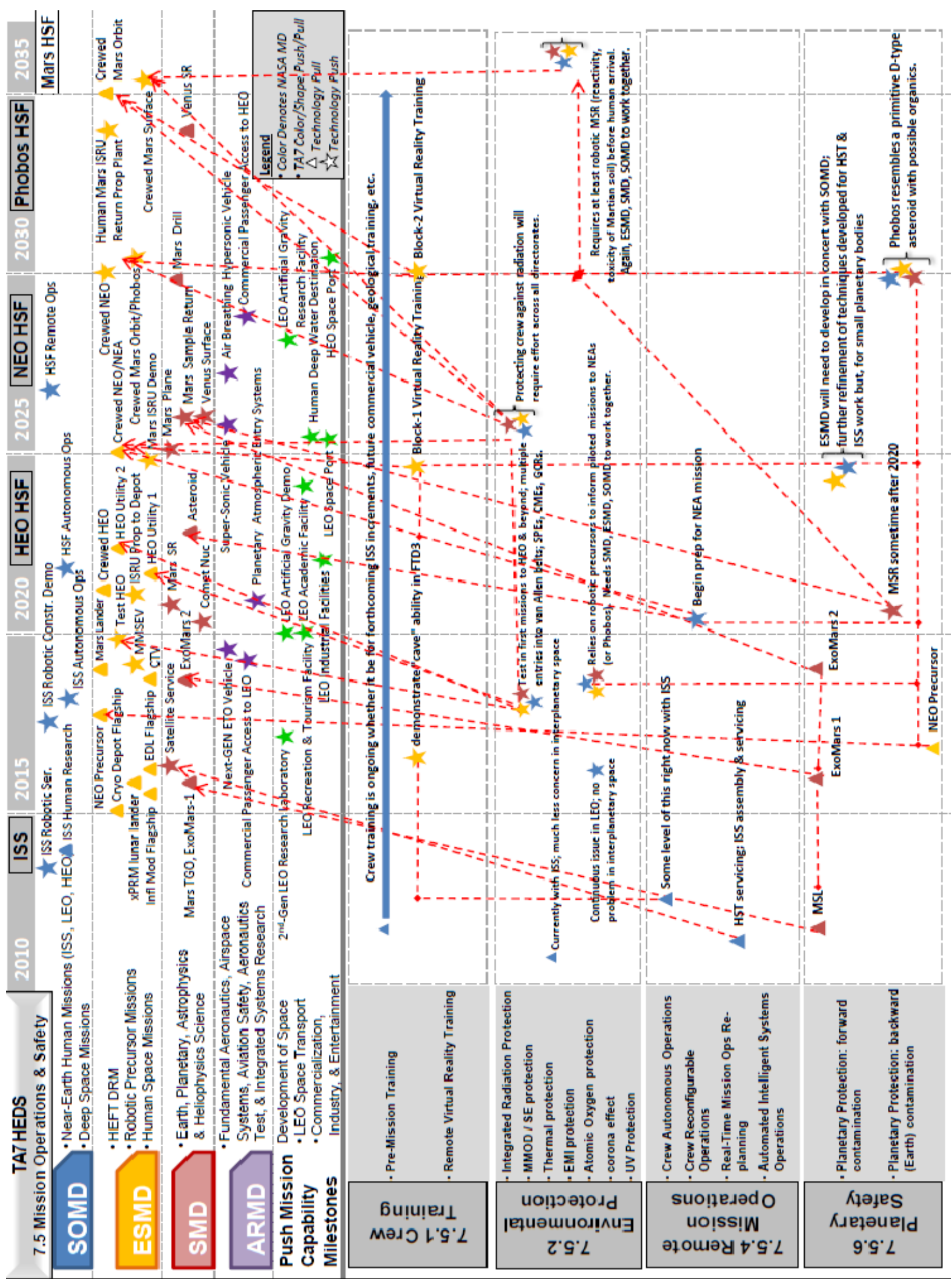

Figure 19: HEDS “Mission Operations and Safety Level-2 Roadmap 


\section{Remote Mission Operations (HEDS TABS 7.5.3)}

The generic Future Crew Exploration Vehicle (FCEV) would have several basic capabilities in order to complete the mission's scientific and technical objectives. These would involve aspects of remote sensing, deployment/redeployment of surface experiment packages, and surface sampling techniques. The precursor mission to the NEO should have adequately characterized the surface and near-space environment to reduce the risk to the FCEV and its assets. Hence, for example, the majority of FCEV operations should take place during close proximity ( a few to several hundred meters from the surface) to the NEO. Such operations have been found to be challenging for remotely controlled spacecraft due to round trip light delay times of several seconds or minutes, but will probably be inconsequential for piloted operations from a vehicle such as a combined FCEV with a modified airlock and inflatable habitation module. In terms of remote sensing capability, the FCEV should have a high-resolution camera for detailed surface characterization and optical navigation. A light detection and ranging (LIDAR) system is necessary for hazard avoidance (during close proximity operations) and detailed topography measurements. In addition, the FCEV might be outfitted with a tunable radar transmitter to perform subsurface tomography of the object. This would allow a detailed examination of the NEO's interior structure. Given that several NEOs appear to have a high degree of porosity (e.g., Itokawa is estimated to be $40 \%$ void space by volume), it is important to measure this characteristic of the target NEO. Such as assessment will not only provide crucial information regarding the formation and impact history of the NEO, but may also have major implications for future hazard mitigation techniques of such objects.

Prior to the crew going out on EVA and interacting with the surface of a small body (NEO/NEA or Phobos), the CEV will also have the capability to place and re-deploy relatively small scientific packages on the surface of the NEO with a significant amount of precision. Packages such as remotely operated (or autonomous) rovers/hoppers could greatly enhance the amount of data obtained from the surface, and rapidly fine tune the site selection for subsequent sample collection. Other packages could contain in-situ experiments designed to test technologies such as surface anchors/tethers, drills/excavation equipment, and materials/component extraction equipment.

To a certain extent, this is currently done on the ISS. The crew has the capability to manually take control over Soyuz, Progress and ATV dockings. Taking this a step further, to tele-robotically operate hardware at NEOs, Phobos, and Martian surface exploration, is not too much of a stretch beyond what is currently accomplished onboard the ISS.

The role of MOD in remote operations will need to change from that with ISS, where ground is in the loop for capabilities from situational awareness to detailed scheduling, as well as real-time telemetry monitoring and commanding. As the round-trip light-time increases to tens of seconds and minutes, along with communication disruptions from occultations and other disruptions, the locus of control will need to shift to autonomous on-board human-machine capabilities with MOD only playing a strategic role. Intelligent software will need to provide situational awareness, close-in (re)scheduling and adaptive planning, diagnostics and prognostics for system health management, co-ordination with robotic assets when the workload is too high for tele-operation, and preliminary science data processing. High-bandwidth communication to Earth, optical or otherwise, will be needed for MOD to be effective even in strategic roles.

\section{Planetary Safety (HEDS TABS 7.5.4)}

While identified here as 'planetary safety,' the more common term used to describe the guiding principle in the design of an interplanetary mission is planetary protection. Planetary protection aims to prevent biological contamination of both the target celestial body (the Moon, Mars, asteroids) as well as backward contamination to the Earth. This principle arises from the scientific need to preserve planetary conditions for future biological and organic constituent exploration - especially when it comes to exobiology and astrobiology. It also aims to protect the Earth and its biosphere from potential extraterrestrial sources of contamination in the event of a sample return mission. The need for planetary protection measures is strongest for missions designed to return a sample of another planet or celestial body to the Earth.

There are five categories of missions to various celestial bodies that are always considered and examined prior to commencing with a mission. Perhaps the most immediately relevant (and recent) are robotic missions to Mars (the Viking landers, the Mars Exploration Rovers (Spirit and Opportunity), Phoenix, and the forthcoming Mars Science Laboratory mission dubbed Curiosity). Spacecraft bound for the Martian surface (as well as comets, primitive asteroids, etc.) must be sterilized before leaving Earth in order to minimize the risk of forward contamination -depositing Earth-originating biological material at the destination. 
Sometime in the next decade (post-2020), there will be missions to return samples from other bodies. For these missions the return vehicle must then be designed such that the sample is returned in highly reliable "bio-container" with measures in place to dispose of any parts of the vehicle that could have been contaminated before re-entry into the Earth's biosphere. This is to avoid backward contamination to the Earth.

Another aspect to planetary safety is planetary defense. Planetary defense are methods by which NEOs might be moved. Piloted missions to NEOs might leave behind ordnance or other impulsive/propulsive devices to perform momentum transfer experiments to the NEO. That information would be applied to future robotic asteroid missions should we discover a NEO on an inbound course towards Earth. Impact events are a fact of life on Earth. NASA could provide humanity few greater legacies than to know the time and place of any cosmic catastrophe, allowing ample time to prepare our response to that inevitable event.

\section{F. Cross-Cutting Systems (HEDS 7.6)}

This section includes systems engineering, technologies for construction, assembly, and deployment of destination systems hardware, and dust mitigation. The Level 3 and 4 breakdown structure is shown in figure 20. The Cross-Cutting Systems Level 2 roadmap shows the detailed fundamental and applied technologies mapped to the pull and push Mission Directorate missions (Fig. 21).

\section{Modeling, Simulations, \& Destination Characterization (HEDS TABS 7.6.1)}

All HEDS systems must operate in non-terrestrial environments that are often difficult to re-create on Earth. Physics-based models and simulation tools will be required to guide technology choices and to predict and verify operation in the actual destination environment. This area includes development and verification of analytical modeling tools and simulation trainers to verify functionality at destination sites and prepare crew and ground staff for human exploration missions. These tools are focused specifically on human destination systems, and will rely heavily on advanced processing capabilities and techniques developed by Technology Area 11: Modeling, Simulation, Information Technology and Processing.

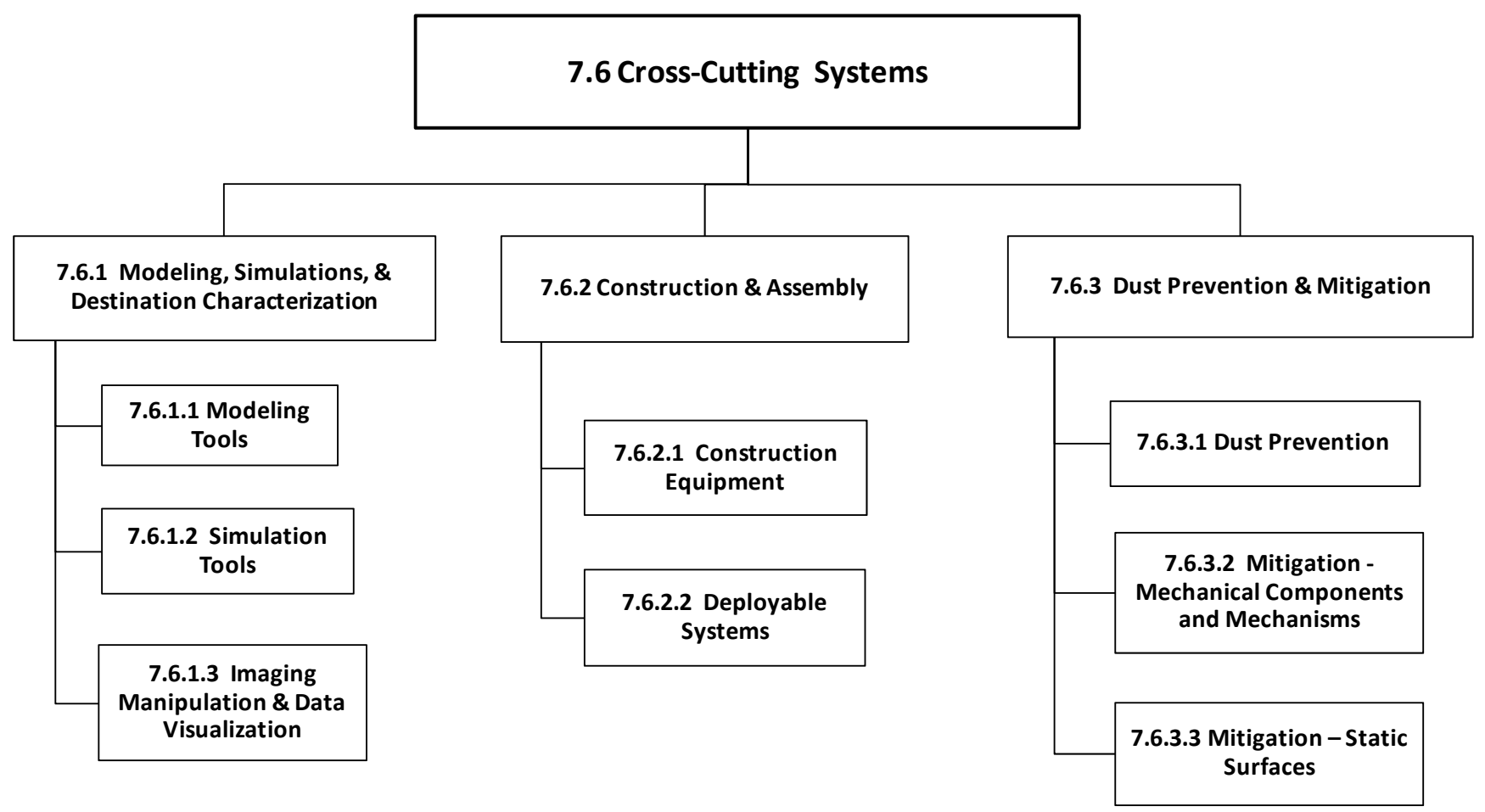

Figure 20: Cross Cutting Systems Level-2 TABS 


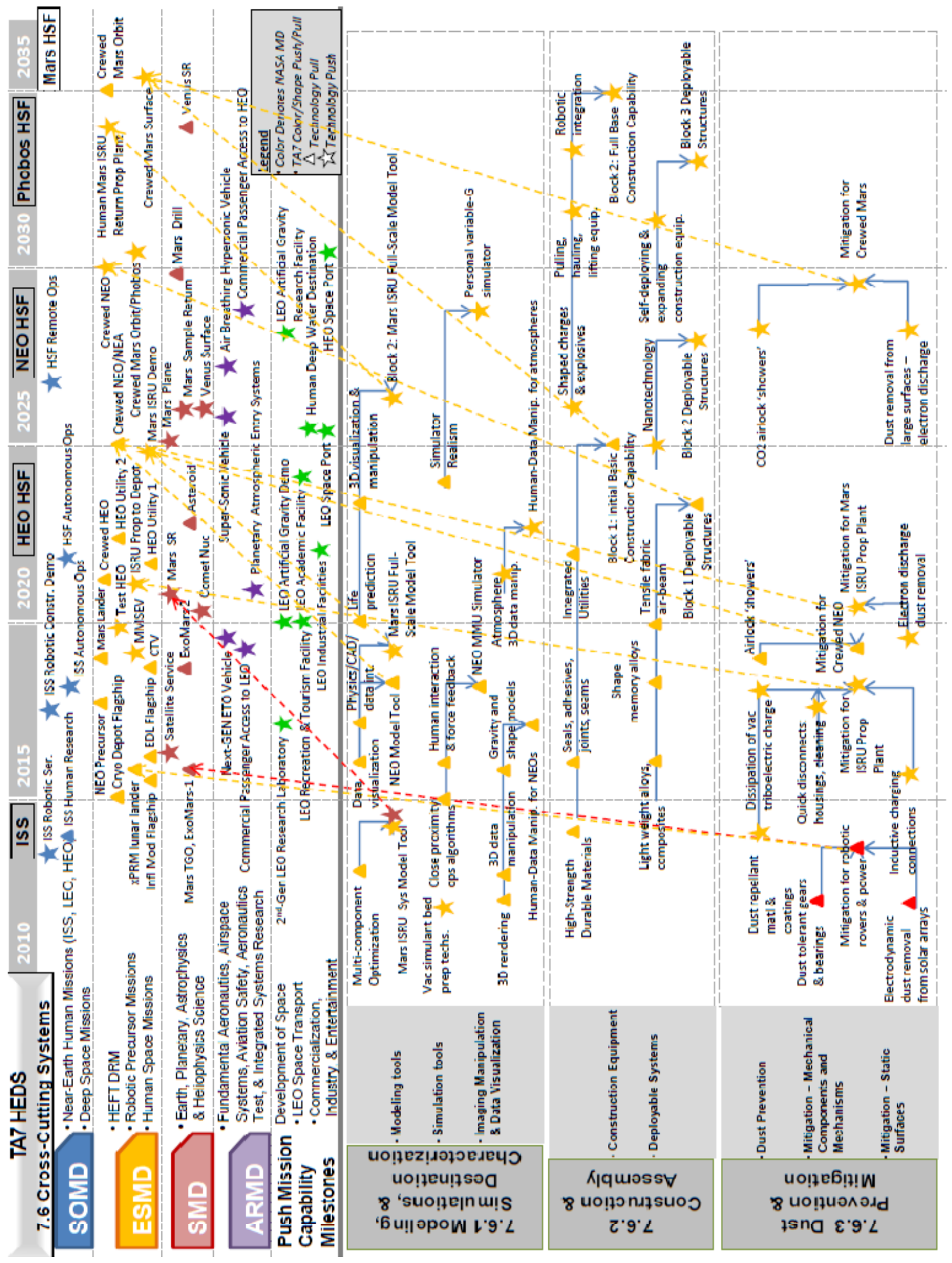

Figure 21: HEDS Cross Cutting Systems Level-2 Roadmap 
While many models already exist for destination system components such as habitats, rovers, and ISRU processing plants, the models are scattered, based in varying computing platforms, and include differing fundamental approaches. In addition, incorporating these models in user-friendly platforms, linking components into flexible system tools, and using the data generated directly in hardware designs all requires additional advancement beyond current practice. Key technical challenges include macro/meso/micro models (e.g., Discrete Element Modeling - DEM) that depict the soil-tool interactions and the soil flows, optimization of discrete and continuous factors across multi-component systems, data visualization for real-time visual results of parametric changes to aid in interpretation, direct model and data integration with CAD and other hardware-focused tools, and life prediction algorithms and models for maintenance, fatigue, wear, life, and performance degradation. Development and validation of physics-based models will reduce DDT\&E costs by allowing fewer build-and-test cycles and coordinated advances in capabilities for system components.

This area includes simulation tools to train astronauts and ground crews for various missions and destinations. Also covered in this area is technology needed to create simulated surface and atmospheric conditions inside test chambers. While the airline and space industries have always relied on sophisticated simulators for training flight and ground crews, several technology advances are required to create more realistic simulations that accurately mimic actual hardware performance and reaction, especially for operations around irregular-shaped orbital bodies (i.e., asteroids) and in off-nominal conditions. A recent front-page article in USA Today (8/31/10) stated that nearly half of all airline fatalities in the last 10 years were partially caused by pilots either being unfamiliar with an offnominal condition or being unprepared in the emergency situation because the actual aircraft behaved more severely than the simulated response. Specific technologies required for developing simulation tools include high-speed processors to increase the amount of fidelity that can be used in the driving algorithms, algorithms to model movement near an irregular-shaped mass, and displays, controls, human-interaction, and force feedback methods to make the simulation more realistic.

Similar to crew training, hardware will need to be tested in simulated environments to demonstrate effective operation in the actual harsh environment. Several technologies are needed before we can set up test chambers that properly simulate the surface and near-surface environment. Specific technologies required for developing simulated environments includes technologies to dry out and prepare simulant beds inside vacuum chambers without disturbing the preparation, vacuum-rated instruments such as bevemeters, cone penetrometers, and moisture sensors to characterize simulant beds, dust tolerant vacuum and roughing pumps, and technologies to suspend dust inside a chamber. These simulated environmental chambers will also be of great value to all other technologies that must operate on the surface of a body or in the dynamic atmosphere above it. Finally, related to both training simulators and environment simulation, better technologies to simulate various gravity levels for humans are required where the gravity level can be controlled instead of the single 'gravity' level of the current underwater training or the very brief $(<1$ minute) periods possible on variable-aircraft flights.

This area also includes technologies for better processing of characterization data to improve the speed and accuracy of data interpretation through manipulation and visualization. A list of specific sensor and instrument needs and their capabilities to gather the necessary data was supplied to TA8: Scientific Instruments, Observatories, and Sensor Systems, and are not covered here. Techniques such as 3D maps, digital elevation modeling, projected images, gravity and shape models, 3D data manipulation, and human-controlled data manipulation will reduce time for data evaluation and increase advanced knowledge of the selected target resulting in better designed flight hardware and reduced mission risk.

\section{Construction \& Assembly (HEDS TABS 7.6.2)}

Because of limitations in launch capability, in-space and surface systems will be launched separately and be assembled in-space or at the destination. This area includes technologies for construction and assembly of in-space and surface structures and completing construction or assembly of deployable systems. Both traditional construction and assembly concepts and advanced deployable systems are covered here.

This area covers construction at the site of pressurized and unpressurized structures for enabling long-duration, deep-space missions and sustained human presence. Applications include habitats, laboratories, unpressurized shelters, under-ground facilities, storage containers or shelters, telescopes, rovers, antennas, hybrid suitlocks, etc. The only existing in-space construction experience comes from assembly of the space station, in which completed components were shipped to orbit and connected to the existing structure through a series of space walks. Recent architecture plans for the lunar base also relied on shipping near-complete units that would require only minimal assembly. With development of advanced technologies, new approaches to design and construction of human systems may be possible to greatly improve mass, power, volume, time, and risk factors. 
Specific technologies in this area include high-strength durable materials; advanced seals, adhesives, and lubricants; packaging technology; integrated utilities; quick disconnects; advanced connection, joining, berthing, and alignment techniques; robotic integration, and nano-enhanced materials. Equipment that must be developed to enable in-space or in-situ construction and assembly includes lifting equipment (pneumatics, cranes, and davits); pushing and pulling equipment; moving and hauling equipment; common chassis designs, and rugged electric motor systems. Civil engineering processes for off-planet destinations are also needed such as shaped charges and explosives; blast debris control; charge optimization and placement; and soil stabilization technology.

This area covers readying and completing construction or assembly of deployable systems such as inflatable solar concentrators, inflatable habitats and labs, storm shelters, and radiation shields. These systems could be partly or wholly provisioned from Earth and then use in-situ resources (regolith, metals, plastics, binders, gases, etc.) to create or complete more rugged, rigid systems and structures capable of withstanding the environment. Potential applications include truss systems, unpressurized shelters, large telescopes, antennas, solar cells, and surfaces garages or bunkers. Specific technologies in this area include pneumatic beams, composite struts, tensile fabric airbeams, light-weight alloys, shape memory alloys, rigidizing materials, and nano-technologies as applied to smart materials.

\section{Dust Prevention \& Mitigation (HEDS TABS 7.6.3)}

The physical and health problems with the lunar dust during the Apollo missions are well documented. By the end of 4 days most of the EVA suits were severely deteriorated and the dust inside the crew capsule was causing serious irritation and health issues. This area covers technologies that will prevent dust from settling on destination systems, or that can remove dust that has accumulated.

Dust prevention includes technologies for preventing dust settling and build-up on components, mechanisms, and surfaces, and in gaseous systems such as airlocks, habitat air, and transfer lines. Specific technologies include dust repellant/dust shedding coatings, dust repellant materials, forced gas showers to decontaminate prior to entering the habitat, $\mathrm{CO} 2$ 'snow' showers (to enhance electrodynamic removal), and dissipation, reduction, and /or elimination of triboelectric charge build-up in a vacuum environment. An additional technology is control and mitigation of dust generation caused by the rocket engine plume. This is covered by TA9: Entry, Descent, and Landing, with some supporting technologies (surface passivation, landing pads) from the ISRU element in this TA.

Dust mitigation includes technologies to remove or tolerate excessive dust build-up on mechanical components and mechanisms and static surfaces such as solar panels, thermal control systems, EVA suits, habitat windows, tools, and electrical and thermal connections. Specific technologies include self-cleaning interface joint seals, advanced bellows for joints, shaft seals, dust-tolerant gears, coatings, and bearings, dust-tolerant greases and lubricants, protective housings and covers for fittings, forced gas cleansing techniques, electrodynamic removal, electron discharge, and 'dust-philic' brush materials and textures.

\section{Path to Technology Readiness Level-Six}

The TA7 team performed a technology readiness level (TRL) assessment of this level-3 technology area. This assessment defined the current stat of the art or practice, current TRL, major challenge(s), milestones and activities to advance to TRL 6 or beyond, key performance parameters, and facilities required to mature to TRL- 6 . The TRL matrix was validated through a series of Technical Interchange Meetings (TIM) with subject matter experts from NASA, academia, industry and other governmental agencies (e.g. DoD, DOE).

\section{Dependencies with Other Technology Areas}

The HEDS TA definition is a mixture of "fundamental" technology development and "applied" technology development._ HEDS has a strong dependency on "basic and fundamental" technology developed by the other Technology Areas that are focusing on discipline areas such as power, propulsion, and life support, for example.

The HEDS TA has dependencies and collaborations with the other 14 TAs. This is inevitable due to the interconnectivities and cross-cutting disciplines ascribed in human space exploration. As long as this relationship is understood, collaboration can and should occur between and among the various technology areas. Collaborations between technology areas can also be understood as: (1) technology development needs being shared by the technology areas and/or (2) possible technology requirements flowing both ways between two technology areas. A good example of collaboration is the wireless/contactless power transfer, where Space Power and Energy Storage Systems TA3 develops the energy storage and generation systems and the Human Exploration Destination Systems 
TA7 develops the wireless/contactless power transfer which must integrate with TA3's energy storage and generation systems.

\section{Benefits to National Needs}

Many of the technologies developed under the HEDS technology area to support human exploration of space will have both direct or spin-off potential to almost all areas of civilized life on Earth. National needs such as manufacturing, energy, health, agriculture, mining, pharmaceuticals, aviation, education, construction, entertainment, environment, and national security will all benefit.

\section{Summary}

The TA7 HEDS Team has identified a diverse portfolio of fundamental and applied technologies that are both evolutionary and revolutionary and capable of sustaining human presence in space at various destinations, thereby enabling NASA's vision and goals of human space exploration. This roadmap lays out a strategic investment that focuses on capabilities based on a five-year cycle of technology maturation and development. Each cycle of technology development (Blocks) provides infusion milestones building upon previous developments that will enhance and enable capabilities in support of "pull" missions while fostering "push" mission opportunities.

The path has been started for the work remains to ensure that this roadmap will provide a clear path to development of advanced technologies to enhance and enable exploration destination capabilities of sending humans into space on sustained missions of exploration and science. Further definition, collaboration, and understanding of current technology developments and gaps are required to bridge future technology requirements and needs. Continued review of Technology Areas roadmaps will be required to refine and maintain NASA's technology future. With a constant pursuit of pushing the envelope of technological advancement, human exploration of space will continue to expand the frontier, engage mankind, and bring benefits to our nation.

\section{References}

1. Airlock, Suitlock, Suit Port Assessment Team Final Report, December 2009

2. Capability Requirements, Analysis, and Integration(CRAI) Overview, NASA Document, 2004 United States Air Force, Chief Scientist (AF/ST), Technology Horizons A Vision for Air Force Science \& Technology During 2010-2030, May 2010

3. Chullen, C. and Westheimer, David T., 2010, "Extravehicular Activity Technology Needs," Paper presented at AIAA Space 2010 Conference, Anaheim, California, August 30-Septemer 2

4. Exploration of Near Earth Objects Objectives Workshop, Summary Report, September 2010

5. Exploration Technology Development Program, Independent TRL assessment, 2009

6. Human Exploration and Development of Space Technology/Commercialization Initiative Strategic Research \& Technology Road Maps, May 2000

7. NASA Early HEFT draft documents

8. Oeftering, Richard C., P. M. Struk, and B. K. Taleghami. A Lunar Surface System Supportability Technology Development Roadmap. AIAA Space 2009 Conference \& Exposition. Sept 14-17, 2009, Pasadena California.

\section{Acknowledgements}

I would like to thank the TA07 Core and Support Team members for all the hard work they have put into this rapid prototyping and aggressively scheduled project. They have done amazing accomplishments with minimal funding under stressful conditions.

I would also like to thank my wife and family for their support in my efforts. 